\title{
Heuristic linear algebraic rank-variance formulation and solution approach for efficient sensor placement
}

\author{
Younghwan Chae, Daniel N. Wilke \\ Centre for Asset and Integrity Management (C-AIM), Department of Mechanical and \\ Aeronautical Engineering, University of Pretoria, Pretoria, South Africa Phone: +2712 \\ 4202432 Fax: +2712 2635087
}

\begin{abstract}
The digital age has significantly impacted our ability to sense our environment and infer the state or status of equipment in our environment from the sensed information. Consequently inferring from a set of observations the causal factors that produced them is known as an inverse problem. In this study the sensed information, a.k.a. sensor measurement variables, is measurable while the inferred information, a.k.a. target variables, is not measurable. The ability to solve an inverse problem depends on the quality of the optimisation approach and the relevance of information used to solve the inverse problem. In this study, we aim to improve the information available to solve an inverse problem by considering the optimal selection of $m$ sensors from $k$ options. This study introduces a heuristic approach to solve the sensor placement optimisation problem which is not to be confused with the required optimisation strategy to solve the inverse problem. The proposed heuristic optimisation approach relies on the rank of the cross-covariance matrix between the observations of the target variables and the observations of the sensor measurement variables obtained from simulations using the computational model of an experiment. In addition, the variance between observations of the sensor measurements is considered. A new formulation, namely the tolerance rank-variance formulation (TRVF) is introduced and investigated numerically on a full field deterioration problem. The full field deterioration is estimated for a plate by resolving a parametrisation of the deterioration field for four scenarios. We demonstrate that the optimal sen-
\end{abstract}

Email address: chyohw97@gmail.com (Younghwan Chae) 
sor locations not only depend on the loading and boundary conditions of the plate but also on the expected ranges for the deterioration parameters. Although the sensor placements are not provably optimal the numerical results clearly indicate computationally efficient near optimal sensor placements.

Keywords: optimal sensor placement, inverse problem, design of experiments, variable reduction, linear algebraic rank, rank tolerance maximisation

\section{Introduction}

Advancements in technology have made sensing accessible for numerous applications that include detection of traffic congestion using GPS sensors (D'Andrea and Marcelloni (2017)), environmental monitoring (Lan, Qilong and Du (2008); Liu et al. (2010)) and human health monitoring (Asada and Reisner (2006)). In structural engineering, experiments are often designed with the aim to characterise a problem e.g. material characterisation (Beilina (2015) or deterioration estimation (Yap (2013) and Mungla, Sharma, and Trivedi (2016)).

This study proposes a new approach to optimise the sensor placements for material characterisation which is an example of an inverse problem in material science. For an inverse problem the sensed information, a.k.a. sensor measurement variables $X$, is practical to measure while the inferred information, a.k.a. target variables $Y$, is either impractical or impossible to measure. In detail, when considering discrete sensor locations, the sensor variables $X$ can be expressed as $X_{n, k}$ where $n$ and $k$, respectively denote the I.D. numbers of observations and locations. Similarly, the target variables $Y$ can be expressed as $Y_{n, p}$ where $n$ and $p$, respectively denote the I.D. numbers of observations and target variables. Note that $X_{n, k}$ is a function of $Y_{n, p}$ for the same observation $n$.

The ability to solve an inverse problem depends on the quality of the optimisation approach and the relevance of information used to solve the inverse problem. In addition, should the inverse problem be ill-posed then multiple solutions may result in the same response with the consequence of small changes in the sensors resulting in significant changes in the inferred information (Hadamard (1902)). The quality of the solution to an inverse problem therefore depends on the following:

1. the relevance of the sensed information, 
2. the quality of the sensed information,

3. the amount of sensed information, and

4. the solution strategy employed to solve the inverse problem.

The relevance and quality of information depend on several factors such as the amount of noise in the measurements and the correlation between the sensor measurement variables $X$ and the target variables $Y$. Lastly, a strategy is employed to solve the inverse problem. Significant research has been done to propose strategies to solve inverse problems (Yao, Sethares and Kammer (1993); Fouladgar (1997)) or to formulate inverse problems for a given arrangement of sensors (Lee and Song (2016)). Strategies to improve the quality of the data being sensed only recently started to gain traction ( $\mathrm{Li}$ et al. (2007); (Krause et al. (2006)), with sensor placement optimisation usually developed for specific applications.

Sensor placement optimisation is a research field that aims to improve the quality and relevance of sensed information when multiple options of where and what to sense are available. Therefore, sensor placement optimisation problems are combinatorial optimisation problems that require $m$ sensors to be selected from $k$ options that make the best data available for a specific application or inverse problem (Korte and Vygen (2012)). Hence, given the measurements of all $k$ sensors, it becomes important to distinguish between the relevance and quality of the various sensors.

In statistical parameter estimation, the relevance of the information is expressed by the estimator-variance (or similarly, mean square error) between the observations of a parameter $\theta$ and its estimator $\hat{\theta}$. By minimising this i.e. computing the maximum likelihood estimator (MLE) (Bishop (2013)) the better the estimator explains the observations. In optimal sensor placement, the $\theta$ equates to the optimal sensor location $X_{k}^{*}$. However, reducing the estimator-variance in statistics is a non-trivial task. The inverse relation of the estimated variance is the Fisher information matrix (FIM) that expresses the amount of information explained for an observation of $Y^{*}$ at a certain point (or equivalently, $Y_{n, 1, \ldots, p}$ ). Hence, the maximisation of reduced scalar representations of the FIM allows for the amount of information that is expressed to be maximised. However, these scalar representations are not unique and may weigh some aspects of the information more than others. Examples include maximising the determinant of the FIM (Isaacs, Klein and Hespanha (2009)) which minimises the estimated uncertainty volume of unbiased $\hat{\theta}$ estimations (Van Trees (1968); Bishop et al. (2007)). The 
minimisation of the trace of the inverse of the FIM, which maximises the independent information is also a popular approach (Achanta, Dasgupta and Ding (2012)). FIM is defined as $J^{T} J$ where $J$ is the Jacobian matrix defined as, $d X_{n}\left(Y^{*}\right) / d X_{k}$ (Frieden (2004); Bishop et al. (2007)), the derivative of the measurement variables $X_{n}$ with respect to the sensor location variables $X_{k}$ when assuming a continuous domain for the sensor locations. Consequently, one of the main issues of relying on FIM is that its information is localised around an observation of $Y^{*}$ (Chisari et al. (2016)). These approaches effectively find the sensor positions $\hat{\theta}$ that gives the highest sensitivity towards the target variables at $Y^{*}$. There has been a number of sensor optimisation approaches proposed that are based on FIM (Li et al. (2007); Isaacs, Klein and Hespanha (2009)) and Gaussian processes (GP) (Wu and Liu (2012); Du et al. (2014, 2015)) to find optimal sensor locations.

The motivation for this study is to develop an alternative sensor optimisation approach that inherently considers spatially distributed variance in a domain around $Y^{*}$ as opposed to the localised variance at $Y^{*}$. This allows us to tailor sensor placement for a more general domain of application as opposed to a specific application. For material characterisation experiments this translates to instrumenting an experimental setup for a specific material (localised) e.g. a steel or for more generally a class of materials (distributed variance) e.g. metals. It has been demonstrated recently that the sensor placement optimisation problem can be expressed by the following three criteria (Chae (2017)), which indicates that sensors should:

1. be correlated to what needs to be inferred,

2. be sensitive to what needs to be inferred, and

3. contribute unique information.

Consider the observations of the sensor measurement variables $X$ and their respective observations of the target variables $Y$ around $Y^{*}$. By increasing the sampling domain around $Y^{*}$ we effectively control the domain of interest. The rank of the cross-covariance matrix $X^{\mathrm{T}} Y$ respectively indicates the collinearity between the observations of $X$ and $Y$. By standardising the observations we avoid biases when variables have significantly different ranges. The Z-score is a standard practice that transforms each variable to the units of standard deviation over the observed sample set. The implication is that the covariance matrix computed using Z-scores is now equivalent to the correlation matrix. Hence, for a given number of variables the larger 
the rank of the cross-correlation matrix the more independent the correlation between the variables i.e. the more unique the information that is being sensed. The consideration of rank has proven difficult as maximising rank is a non-convex optimisation problem that has been optimised using genetic algorithms (Wang, Liu and Liu (2014)). However, recent approaches have been proposed to avoid this difficult problem by transforming the problem to be convex. This is done by introducing a related objective function, or a so-called proxy objective function (Joshi and Boyd (2009)). The nuclear norm has proven to be a popular choice to convexify the rank maximisation problem (Recht, Fazel and Parrilo (2010)), however, the validity of solving this problem in relation to the original problem has been questioned (Dai and $\mathrm{Li}$ (2014)). As will be discussed in the next section we can maximise linear independence by considering the tolerance related to the definition of rank which is the approach we follow in this study.

A second consideration is that noise in the sensor measurements is inevitable in reality. Thus, it is mandatory to maximise the signal to noise ratio (SNR). This is done by maximising the measurement variance $\sigma_{X_{n}}^{2}$ over the observations of $X$, which is not to be confused with the estimator-variance mentioned earlier for FIM (or the variance in the sensor locations $\sigma_{X_{k}}^{2}$ ). This study introduces a new formulation to solve the sensor placement optimisation problem, namely the tolerance rank-variance formulation (TRVF). This approach maximises the bi-objective optimisation problem of maximising the observation variance of the measurement variables $\sigma_{X_{n}}^{2}$ and maximising the tolerance of the linear algebraic rank of the cross-covariance matrix.

Lastly, a new heuristic approach for solving the optimisation problem is proposed in Section 3, which we refer to as sequential sensor placement (SSP), in which we add sensors sequentially as opposed to solving the intractable exhaustive combinatorial optimisation problem. Although we present no formal proof of optimality, numerical results do indicate that quality solutions are obtained computationally efficiently.

To summarise, the contributions of this paper include:

1. a bi-objective formulation based of variance and rank tolerance to solve the sensor placement optimisation problem is proposed,

2. for the bi-objective formulation the parameter choice of $\alpha=0.5$ is shown to perform effectively and robustly on four inverse problems,

3. the formulation allows for a simplistic but efficient strategy to the define domain of interest for the inverse problem for which the sensors are to 
be placed,

4. a heuristic approach to solve the proposed sensor optimisation problem is proposed that is demonstrated to result in quality solutions.

\section{Tolerance Rank-Variance Formulation (TRVF)}

The tolerance rank-variance formulation (TRVF) is defined as a bi-objective optimisation problem. Numerous strategies are available to solve a bi-objective optimisation problem that include determining the Pareto front using evolutionary strategies designed to find the Pareto front even when it is not convex e.g. multi-objective particle swarm optimisation (MOPSO) and multiobjective genetic algorithm (MOGA) (Reyes-Sierra and Coello Coello (2006); Konak, Coit and Smith (2006)). In this study to simplify the bi-objective formulation, we write the bi-objective problem as a weighted sum that requires a parameter $\alpha$ to be chosen. Although the weighted sum approach simplifies the problem it has two shortcomings that need to be mentioned. It can only recover a convex part of the Pareto front and the distribution of design along the Pareto front may vary significantly. We show that a choice of $\alpha=0.5$ performs well on all the problems considered in this study.

The first objective is to maximise the tolerance of the linear algebraic rank of the covariance matrix $X^{\mathrm{T}} Y$ between the observations of the measurement $X$ and target variables $Y$. A large tolerance for a full rank system indicates that the linear equations which represent the correlation between the measurement and target variable observations are distinct and unique resulting in less overlap between the explanatory domains. The rank tolerance objective is to find the set of sensors $X_{C}$ that

$$
\max _{X_{C}} \epsilon_{r}
$$

such that

$$
\max _{\epsilon_{r}} \operatorname{rank}\left(\left(X_{C, Z}^{T} Y_{Z}\right), \epsilon_{r}\right)=\min (n, C, p),
$$

where $\epsilon_{r}, X_{C, Z}$ and $Y_{Z}$ respectively denote the rank tolerance parameter, the sensor measurement variables at the chosen (indicated by subscript $C$ ) locations $X_{C}$ and the resulting target variable observations $Y$. The observations of both variables are Z-scored (Kreyszig (1979)) as indicated by the subscript $Z$. The Z-score standardises the observations of a variable $X$ as follows

$$
X_{Z}=\left[\frac{X_{n, 1}-\bar{X}_{n, 1}}{\sigma_{X_{n, 1}}}, \ldots, \frac{X_{n, k}-\bar{X}_{n, k}}{\sigma_{X_{n, k}}}\right],
$$


where the superscript bar $(\bar{\cdot})$ and $\sigma$ denote the mean and standard deviation for the observations of variable $X$. By adjusting the rank tolerance given by (1) we can control the required amount of the independence between the variables.

A second objective is required to ensure that the most sensitive sensors are selected i.e. sensors with high variance, as sensors that maximise the uniqueness do not necessarily maximise their sensitivity. Sensors with higher variance allows for larger signal to noise ratio (SNR) making the inverse problem less susceptible to aleatory uncertainties, particularly in cases where the aleatory uncertainties are common to all signals for a specific sensor type referred to as class noise. In turn, when aleatory uncertainties can predominately be expressed as a fraction of the measured variance of a single sensor, point noise, the second objective is less important. In practice, the aleatory uncertainties are the result of both class noise and point noise with class noise requiring a minimum SNR to be satisfied (Chae (2017)). For a chosen set of sensors $X_{C}$ the aim is to find the maximum of the product of variances of the various sensors expressed by

$$
\sigma_{X_{n}}^{2}=\prod_{i=1}^{N_{\text {sensor }}} \sigma_{X_{C, n, i}}^{2},
$$

where the product ensures that all sensors contribute variance towards the cost function with similar order of magnitude, as any sensor with low or near zero variance would penalise the product significantly even at the benefit of three or four times higher variance in another sensor. The benefit of this towards the SNR is that all sensors have similar variance, and consequently have similar robustness towards noise in the signals.

The bi-objective optimisation problem consisting of (1) and (3) requires us to find the set of sensors that maximises both the rank tolerance $\epsilon_{r}$ and variance $\sigma_{X}^{2}$. Although various strategies are available to solve for the general case of non-convex Pareto fronts, we limit ourselves to the weighted sum approach in this study that implies we restrict ourselves to only a convex part of the Pareto front for the bi-objective optimisation problem. Given $j$ options to add sensors, we first compute $L_{\epsilon_{r}}\left(X_{C}\right)$ and $L_{\sigma_{X_{n}}^{2}}\left(X_{C}\right)$ for each sensor option to construct a rank tolerance and variance list. We then normalise each list by its respective maximums. A parameter $\alpha$ between 0 and 1 is introduced for the weighted sum approach as follows

$$
\max _{X_{C}} f\left(X_{C}\right)=\alpha L_{\epsilon_{r}}\left(X_{C}\right)+(1-\alpha) L_{\sigma_{X_{n}}^{2}}\left(X_{C}\right),
$$


where $\alpha=1$ recovers a rank only solution, and $\alpha=0$ a variance only solution. Computing TRVF for the full combinatorial problem is computationally intractable and hence we require some tractable solution strategy to solve TRVF.

\section{Strategies to solve TRVF}

A strategy, namely sequential sensor placement (SSP), to solve the tolerance rank-variance formulation (TRVF) is proposed in this study. SSP places one sensor at a time up to the desired number of sensors $N_{m}$ by optimising Eq.(4) for each additionally placed sensor and keeping the placed sensors the same. It does not guarantee optimal solutions but requires much less computational time than the conventional exhaustive combinatorial approach, which makes SSP computationally tractable for practical problems.

For example finding the optimum by considering all combinatorial solutions exhaustively (Korte and Vygen (2012)) requires

$$
\left(\begin{array}{c}
N_{k} \\
N_{m}
\end{array}\right)=\frac{N_{k} !}{N_{m} !\left(N_{k}-N_{m}\right) !},
$$

combinations to be checked, which for all practical purposes is intractable.

\section{Structural health monitoring problem}

Structural health monitoring (SHM) is becoming increasingly important with ageing infrastructures as well the exposure to significant environmental loading conditions to properly assess a structures life cycle. This allows for the proper management and life extension that may have significant financial implications. SHM is a challenging problem in which ideally the full deterioration field of a structure needs to be recovered, which may require significant sensing infrastructure to sense sufficiently. Consequently, allowing for the optimal placement of sensors may significantly alleviate the strain placed on a sensing infrastructure. As a result, to investigate the performance of TRVF, a recently proposed structural deterioration inverse problem by Lee and Song (2016), is considered.

This inverse problem aims to recover the spatial parametrisation for Young's modulus, $E(x, y)$. Lee and Song (2016) proposed an algorithm that improves the quality of solving inverse problems by combining Bayesian 
networks (BN) and maximum likelihood estimation (MLE) instead of conventional ordinary least squares (Lee and Song (2016)). In this study we demonstrate that inverse problems can be improved by optimising the placement of the sensors to improve the quality of the available information. We obtained all required information to exactly reproduce the problem proposed by Lee and Song (2016) by direct correspondence with the authors.

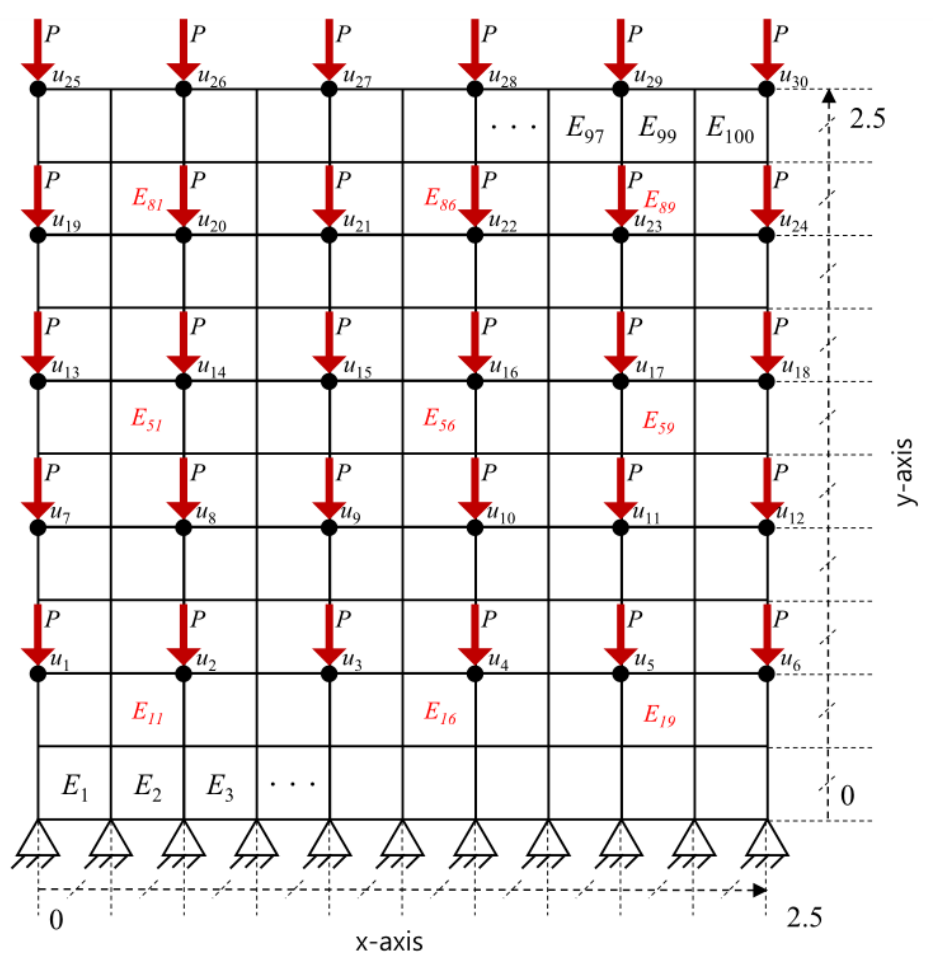

Figure 1: Diagram of the plate, loads and boundary conditions used in our deterioration study (Lee and Song (2016)).

The structural model of the problem proposed by Lee and Song (2016) is depicted in Figure 1 . The model of $2.5 \mathrm{~m} \times 2.5 \mathrm{~m} \times 1 \mathrm{~m}$ is constructed by $10 \times 10$ plane stress Q4 elements that are fully integrated. The material parameters include Poison ratio of 0.3 and a spatial mean $\mu_{E}$ of $200 \mathrm{GPa}$ for the spatially varying $E(x, y)$, with the real numbers $x \in[0,2.5]$ and $y \in[0,2.5]$. The variation in $E(x, y)$ due to the deterioration of the plate as expressed by

$$
E(x, y ; \mathbf{w})=\mu_{E}(x, y)\left(1-f_{E}(x, y ; \mathbf{w})\right),
$$


with the deterioration field function

$$
\begin{aligned}
f_{E}(x, y ; \mathbf{w})= & \Delta E \cdot \exp \left(-\frac{1}{2(1-\rho)}\left[\frac{\left(x-m_{x}\right)^{2}}{s_{x}^{2}}+\ldots\right.\right. \\
& \left.\left.\frac{\left(y-m_{y}\right)^{2}}{s_{y}^{2}}-\frac{2 r\left(x-m_{x}\right)\left(y-m_{y}\right)}{s_{x} s_{y}}\right]\right)
\end{aligned}
$$

where the six parameters

$$
\mathbf{w}=\left(m_{x}, m_{y}, s_{x}, s_{y}, \rho, \Delta E\right),
$$

defines a specific deterioration field. The upper and lower bounds that define the ranges for each of the six parameters of $\mathbf{w}$ when solving the inverse problem are

1. centre in $x$ given by $m_{x} \in[0.125,2.375]$,

2 . centre in $y$ given by $m_{y} \in[0.125,2.375]$,

3. scaling parameter given by $\rho \in[-0.95,0.95]$,

4. variance in $x$ given by $s_{x} \in[0,1]$,

5. variance in $y$ given by $s_{y} \in[0,1]$, and

6. change in Young's modulus given by $\Delta E \in[0,0.9]$.

The inverse problem error function $\mathbb{E}(\mathbf{w})$ to be minimised computes the sum of the absolute difference between the expected $u_{y, e}$ and measured $u_{y, m}$ displacements in the $\mathrm{y}$-direction and is given by

$$
\mathbb{E}(\mathbf{w})=\sum_{i=1}^{30}\left(\left|u_{y, e, i}(\mathbf{w})-u_{y, m, i}(\mathbf{w})\right|\right) .
$$

In addition, the measured displacements $u_{y, m}$ are modelled to contain noise, namely, 1\%, 5\%, 10\% zero-mean normally distributed random point noise that is relative to the magnitude of the measurements.

The plate is clamped at the bottom which reduces the free degrees of freedom to 110 free DOFs $(10 \times 11)$. A static force of $10 \mathrm{GN}$ is applied downward as indicated by the nodes with arrows in Figure 1. A total number of 30 sensors which only measure displacement in the y-direction $u_{y}$ are originally placed at each node where a force is applied as shown in Figure 1. In this study the placement of the sensors will be optimised for each of the four scenarios using SSP to solve TRVF. The four deterioration fields 
Table 1: Four deterioration fields defined by four choices for $\mathbf{w}$ presented as Scenario 1 to 4 .

\begin{tabular}{lllllll}
\hline Scenarios & $m_{x}$ & $m_{y}$ & $\rho$ & $s_{x}$ & $s_{y}$ & $\Delta E$ \\
\hline Scenario 1 & 1.125 & 1.125 & 0 & 0.9 & 0.9 & 0.85 \\
Scenario 2 & 1.125 & 1.125 & 0.95 & 0.9 & 0.9 & 0.85 \\
Scenario 3 & 0.125 & 0.125 & 0 & 0.7 & 0.7 & 0.85 \\
Scenario 4 & 2.375 & 2.375 & 0 & 0.7 & 0.7 & 0.85 \\
\hline
\end{tabular}

considered in this study, that resemble four deterioration scenarios, are listed in Table 1. These four scenarios represent four spatial deterioration fields that are spatially distinct and that may typically result in different parts of a structure. Scenario 1 presents isotropic deterioration that is concentric to the centre of the plate; Scenario 2 anisotropic deterioration across the diagonal of the plate. Scenarios 3 and 4 present foundation or support deterioration at respectively the lower left and upper right corners of the plate. All four scenarios are considered in detail in Section 5.

\subsection{Optimising the TRVF for Optimal Sensor Placement}

To optimise the sensor placement by solving the TRVF, we first create the finite element model as discussed in the previous section. As discussed the deterioration field is determined by six parameters $\mathbf{w}$. Sensors can be placed to recover a specific deterioration field (specialised application) or a set of deterioration fields (more general application). Since there is always some uncertainty regarding the set of deterioration fields a sensor layout should recover, we consider that each parameter has a confidence interval of $15 \%$ of the defined range of a parameter i.e. a $30 \%$ variation around the parameter values for a scenario when sampling the datasets. This subspace was sampled using Latin Hypercube Sampling (LHS) (Tang (1993); Helton, Davis, and Johnson (2005)) using $n=300$ samples. Hence, the dimensions of $X$ are $300 \times 110$ and the dimensions of $Y$ are $300 \times 6$ for the six parameters $\mathbf{w}$. Having constructed the datasets $X$ and $Y$, we can proceed to consider solving TRVF. For the bi-objective parameter $\alpha$ in Eq. 4, we consider $\alpha=0,0.5,1$. This simple choice of $\alpha=0.5$ is made to demonstrate that the proposed approach performs robustly and efficiently without requiring any tweaking on four different problems. In addition, $\alpha$ is chosen as $\alpha=0$ and $\alpha=1$ to clearly demonstrate that both criteria in the multi-objective formulation 
indeed matter. As we only consider these three choices for $\alpha$ in this study, a future study is warranted to properly investigate the effect of other choices for $\alpha$ as well as other approaches to recover the full Pareto front as opposed to only the convex part thereof.

Optimally selecting 30 sensors from 110 possible locations exhaustively requires $8.3662 \times 10^{26}$ combinations to be evaluated whereas SSP only requires 2865 sensors $(=110+109+\ldots+81)$ to be tested. This only took 1.387 seconds using a single core from 'Intel(R) Core(TM) i7-4702MQ CPU @ 2.20GHz, 2195Mhz, 4 cores, 8 logical processors. Although SSP does not guarantee the optimal sensor set it does offer quality solutions for the problems considered in Section 5.

Once the optimal sensors have been selected they can be used to solve the inverse problem in order to quantify their performance. The finite elementupdated inverse problem is solved using Matlab (2015)'s interior-point algorithm with bound constraints to minimise Eq. 9. The measured displacement $u_{y, m}$ contains normally distributed point noise for which its magnitude is relative to either $1 \%, 5 \%$ or $10 \%$ of the magnitude of the measurement. The estimated parameters $\mathbf{w}$ were computed as the average values of results from 50 solutions to the inverse problem using different initial starting guesses. The initial guesses for $\mathbf{w}$ were bound by the upper and lower limits defined for every parameter, except for $\rho$ which was bound between 0 and 0.1 instead of -0.95 and 0.95 . The optimised and original sensor layouts were all subjected to the identical stochastic noise over all the runs as well as the same initial starting points. The optimisation algorithms were run until convergence but the results after 15 iterations are also reported to allow for an intermediate comparison between the sensor layouts to identify the primary drivers for initial progress.

\section{Numerical Results}

\subsection{Scenario 1}

The shape of the deterioration field for Scenario 1 is concentric around the centre of the plate as shown in Figures 2(a)-(d). The optimised sensor locations are indicated as red dots for choices of $\alpha=1,0.5,0$ respectively in Figures 2(a)-(c), while the originally placed sensor locations by Lee and Song (2016) are indicated in Figure 2(d). The results are for 1\% noise added to the sensor measurement and only after 15 iterations of solving the inverse problem. 
It is evident from Figure 2(c) that maximising variance on its own does not perform well with the sensors clustering around the edges of the top of the plate. Ideally, all sensors would like to be at the same location of maximum variance but since sensor locations are enforced to be distinct, they cluster spatially at the domains of high variance in vertical displacement.

Considering only uniqueness of information in the bi-objective with $\alpha=1$ as depicted in Figure 2(a) resulted in the best initial performance (after 15 iterations) with a similar sensor layout to the $\alpha=0.5$ solution depicted in Figure 2(b). It is evident that uniqueness of information results in placing sensors that are more spatially distinct as information of each sensor is enforced to be distinct from previously added sensors. The sensors are placed around the centre and bottom of the plate.

Solutions for $\alpha=0.5$ and $\alpha=1$ both outperform the originally placed sensors by Lee and Song (2016) as quantified in Table 2. Note that Table 2 reports the number of iterations (\# iter.), function evaluations (\# f.e.) and difference norm $(\|\mathbf{F}(E)\|)$ computed between the predicted $E(x, y)$ and known $E(x, y)$ on a $100 \times 100$ spatial grid. This is estimated for the various sensor placements using three levels of noise for Scenario 1. Considering the converged optimisation results as opposed to only after 15 iterations it is evident that both uniqueness of information and variance $(\alpha=0.5)$ is critical to consider, outperforming all solutions for all levels of noise. The $\alpha=0.5$ solution outperforms the next best solution by more than a factor of 3 for $1 \%$ noise. This also implies that both uniqueness of information and variance drive the solution of the inverse problem towards the end of an optimisation run. As expected the difference between optimised and originally placed sensors decreases as the noise increases, as it is clear that all sensor layouts are equal when only noise is present.

Figures 3 and 4 depict the $E(x, y)$ contours for various amounts of noise when solving TRVF using $\alpha=0.5$ and the original sensor layout (Lee and Song (2016)) after 15 iterations and for the converged solutions, respectively. When only 15 iterations are allowed for the inverse problems, Figure 3 shows that the estimated solutions are far from the exact solutions for both the TRVF sensor layout and the original sensor layout whereas the converged optimised results, depicted in (Figure 4, clearly indicate that TRVF sensor layout outperforms the original sensor placement of Lee and Song (2016). We stress here that Lee and Song (2016) did not consider optimising the sensor layout and we merely quantify in this study the improvements that can be made when this is considered. 
Table 2: Scenario 1: inverse problem solutions using the sensor layouts for TRVF for $\alpha=1,0.5$ and 0 against the original sensor placement for different levels of stochastic noise.

\begin{tabular}{|c|c|c|c|c|c|c|c|c|c|}
\hline \multirow[b]{2}{*}{ Noise } & \multirow[b]{2}{*}{ Method } & \multicolumn{4}{|c|}{ Initial optimisation performance (after 15 iter.) } & \multicolumn{4}{|c|}{ Converged optimisation performance } \\
\hline & & \# iter. & \# f.e. & $\|\mathbf{F}(E)\|$ & $\%$ f.v. diff. & \# iter. & \# f.e. & $\|\mathbf{F}(E)\|$ & $\%$ f.v. diff \\
\hline \multirow{4}{*}{$1 \%$} & $\alpha=1$ & 15 & 125.3 & 356.9 & -92.6 & 72.2 & 700.3 & 60.1 & -97.5 \\
\hline & $\alpha=0.5$ & 15 & 134.1 & 496.9 & -80.7 & 79 & 765.8 & 18.7 & -95.8 \\
\hline & $\alpha=0$ & 15 & 131.1 & 1667.8 & -96 & 104.3 & 931.5 & 158.6 & -97.8 \\
\hline & Original & 15 & 129.1 & 744.6 & -78.1 & 80.1 & 773.3 & 60.7 & -96 \\
\hline \multirow{4}{*}{$5 \%$} & $\alpha=1$ & 15 & 126.4 & 512.8 & -84.5 & 86.2 & 825.7 & 261.8 & -87.4 \\
\hline & $\alpha=0.5$ & 15 & 133.7 & 841.1 & -68.2 & 96.3 & 922.4 & 90.2 & -80.1 \\
\hline & $\alpha=0$ & 15 & 127.3 & 3768.5 & -84.2 & 104.8 & 939 & 775.9 & -88.6 \\
\hline & Original & 15 & 128.5 & 484.9 & -72.5 & 85 & 812.9 & 253.2 & -80.4 \\
\hline \multirow{4}{*}{$10 \%$} & $\alpha=1$ & 15 & 125.5 & 624.8 & -72.7 & 82.5 & 790.8 & 455 & -75.7 \\
\hline & $\alpha=0.5$ & 15 & 132 & 788.5 & -57.3 & 87 & 839.8 & 223.3 & -64.1 \\
\hline & $\alpha=0$ & 15 & 130.6 & 3300 & -74 & 135.1 & 1172.8 & 1196.3 & -76.3 \\
\hline & Original & 15 & 128.4 & 811.3 & -57.7 & 95.9 & 905.7 & 378 & -64 \\
\hline
\end{tabular}

Table 3 quantifies the differences in the optimal parameter values w when solving the inverse problem using the TRVF sensor layout and the original sensor layout by Lee and Song (2016) to the actual parameter values for the parametrisation of $E(x, y)$ as tabulated in Table 1 . This effectively quantifies the uniqueness of the parametrisation of $E(x, y)$ and allows for a comparison between the different solutions. Ultimately, the errors as tabulated in Table 2 are of primary concern. Although the overall errors $\|\mathbf{F}(E)\|$ for TRVF $(\alpha=$ 0 ) are less than those for the original sensor layout, each individual parameter error for TRVF can be sometimes greater than that for the original sensor layout as shown in Table 3.

\subsubsection{Scenario 2}

The shape of the deterioration field for Scenario 2 is a diagonal shape as shown in Figures 5(a)-(d). The optimised sensor locations are indicated as red dots for choices of $\alpha=1,0.5,0$ respectively in Figures 5(a)-(c), while the originally placed sensor locations by Lee and Song (2016) are indicated in Figure 5(d). The results are for $1 \%$ noise added to the sensor measurement only after 15 iterations of solving the inverse problem. It is evident from Figure 5(c) that maximising variance on its own does not perform well with the sensors clustering around the edges of the top of the plate. Considering only 


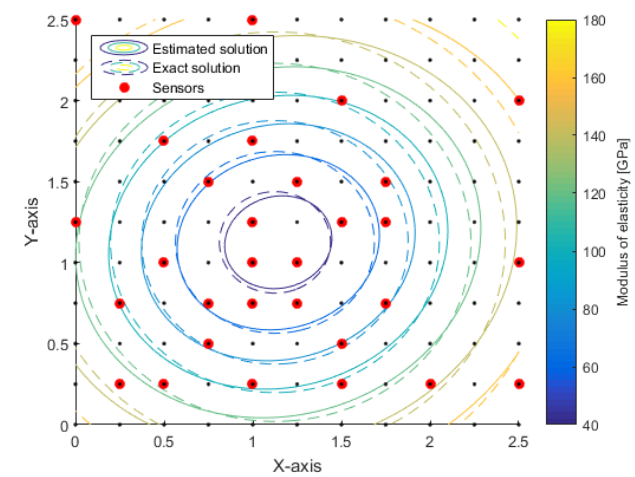

(a) TRVF $\alpha=1$

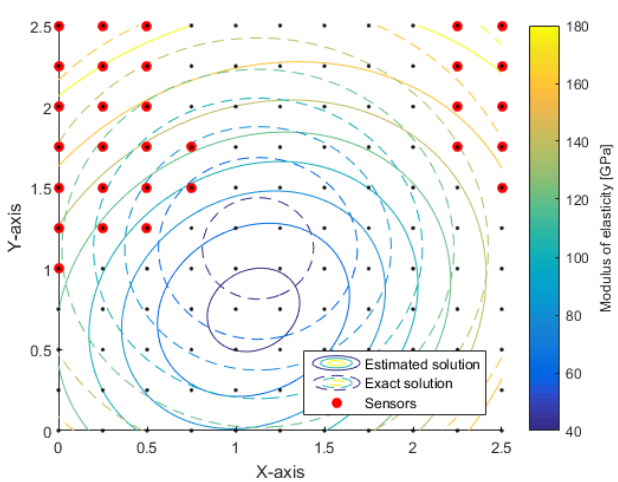

(c) TRVF $\alpha=0$

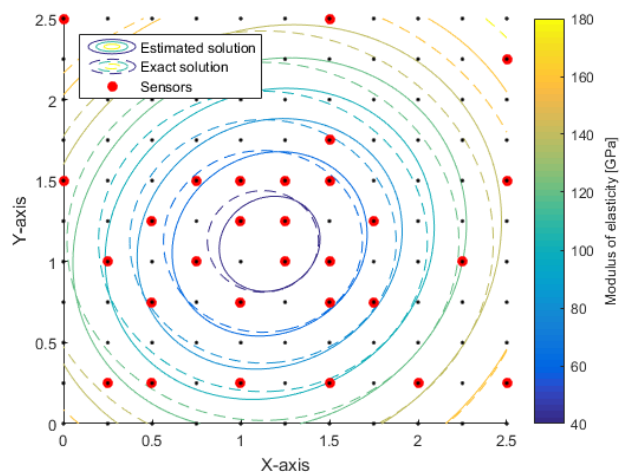

(b) TRVF $\alpha=0.5$

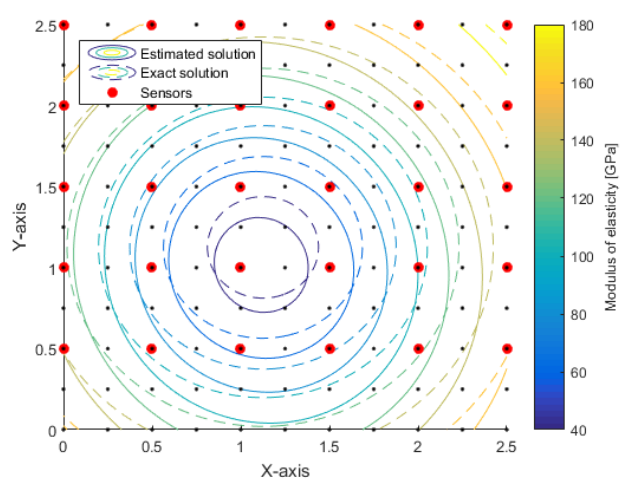

(d) Original sensor placements

Figure 2: Scenario 1: optimal sensor locations obtained by solving TRVF $(\alpha=1,0.5,0)$ presented respectively in (a),(b) and (c). In addition, (d) the sensor placement used by Lee and Song (2016). Depicted is the solution after 15 iterations of solving the inverse problem to characterise the deterioration contours of $E(x, y)$ with $1 \%$ of point noise on the measurements. 


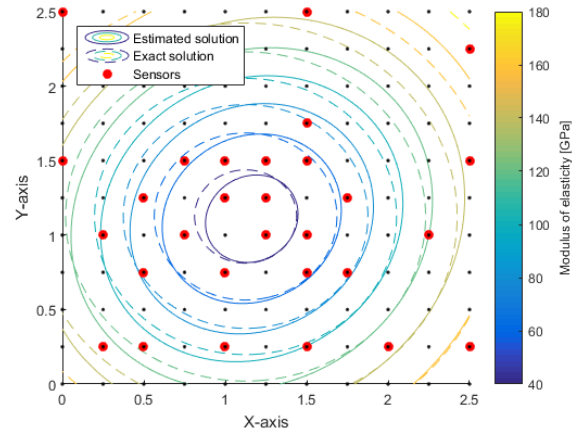

(a) Optimised sensors with $1 \%$ noise

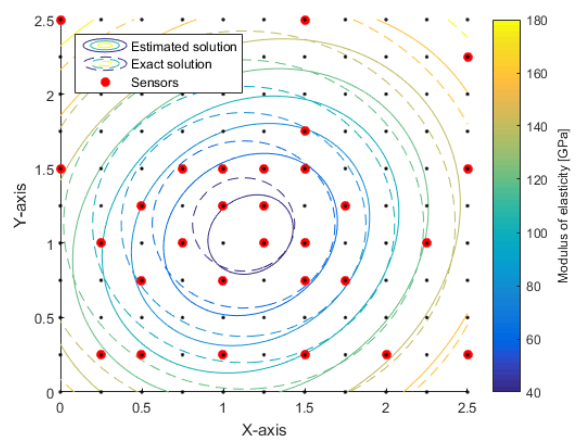

(c) Optimised sensors with $5 \%$ noise

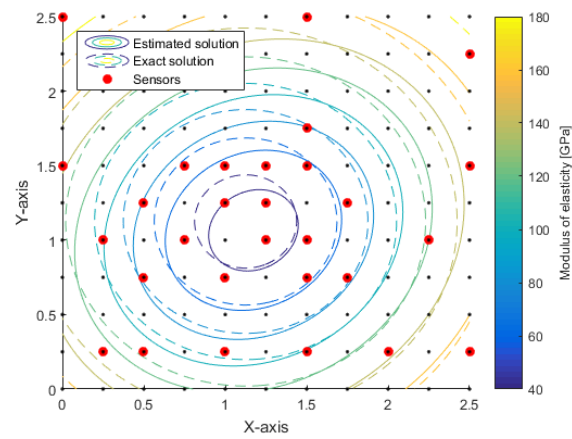

(e) Optimised sensors with $10 \%$ noise

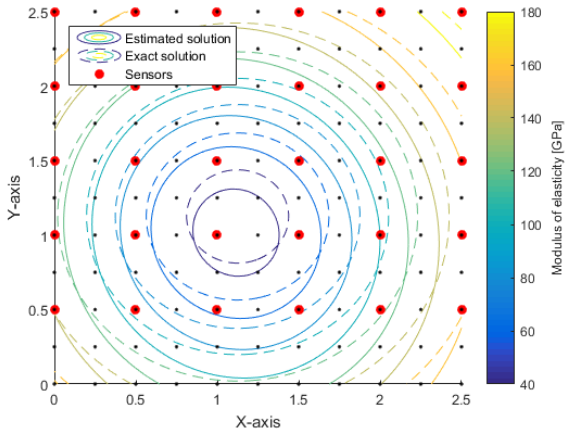

(b) Original sensors with $1 \%$ noise

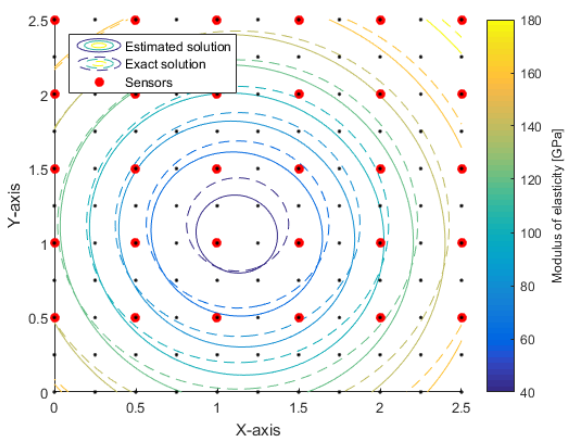

(d) Original sensors with $5 \%$ noise

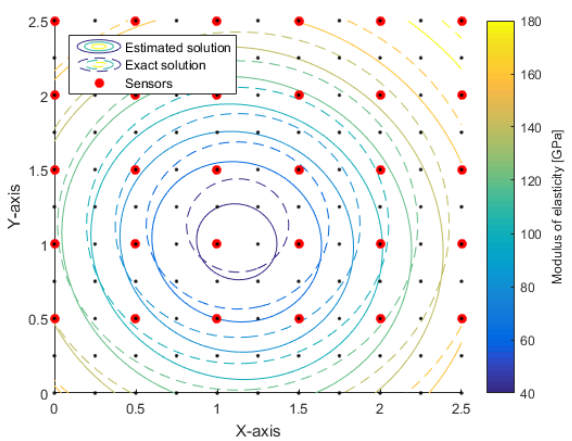

(f) Original sensors with $10 \%$ noise

Figure 3: Scenario 1: optimal deterioration contours of $E(x, y)$ after solving the inverse problem to convergence using the optimal sensor placement obtained by solving TRVF ( $\alpha$ $=0.5$ ) versus the sensor placement used by Lee and Song (2016) with (a),(b) 1\%, (c),(d) $5 \%$ and (e),(f) $10 \%$ point noise on the measurements. 


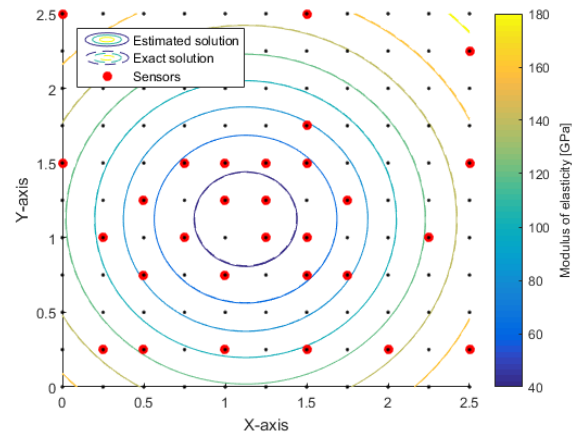

(a) Optimised sensors with $1 \%$ noise

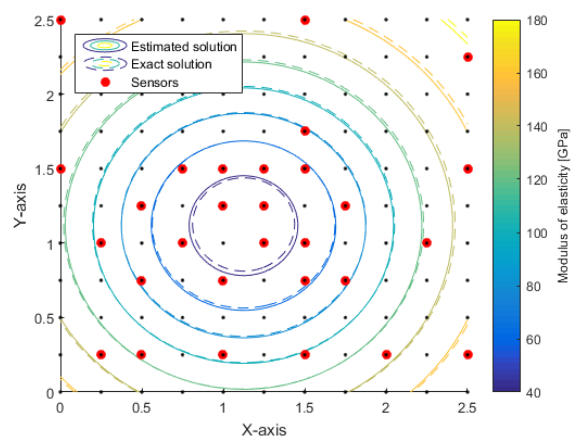

(c) Optimised sensors with $5 \%$ noise

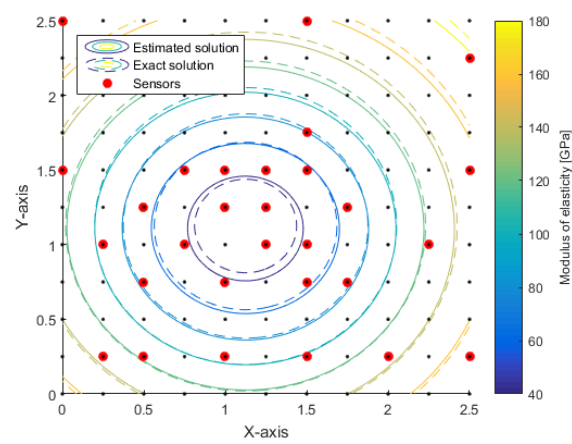

(e) Optimised sensors with $10 \%$ noise

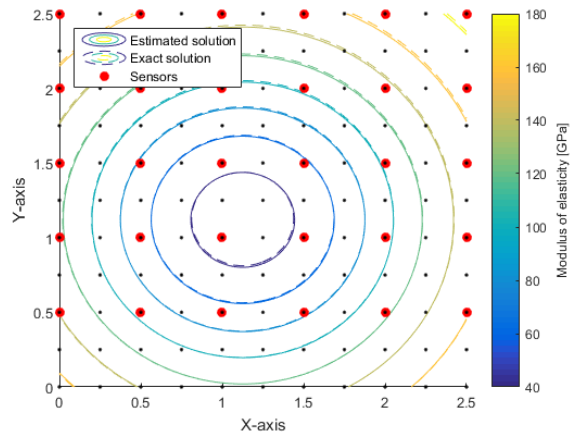

(b) Original sensors with $1 \%$ noise

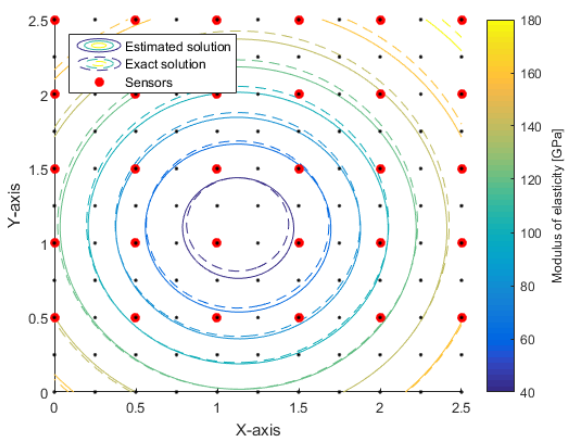

(d) Original sensors with 5\% noise

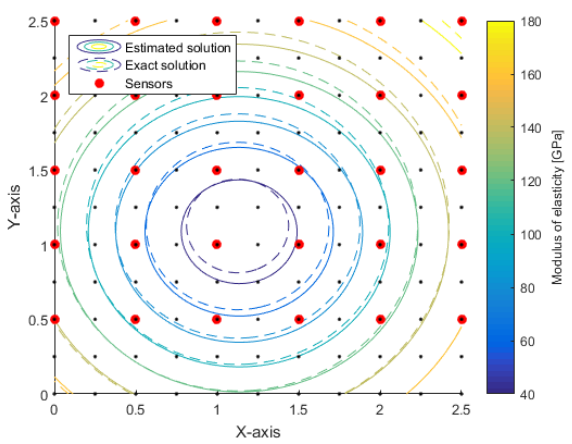

(f) Original sensors with $10 \%$ noise

Figure 4: Scenario 1: comparison between the deterioration contours of $E(x, y)$ recovered using TRVF $(\alpha=0.5)$ versus the original sensor placements for the converged optimisation results using various amounts of relative noise. 
Table 3: Scenario 1: parameter errors for TRVF $(\alpha=0.5)$ and the original sensor placement by Lee and Song (2016) against the known solution as tabulated in Table 1.

\begin{tabular}{llllllll}
\hline Scenario 1 & & $m_{x}$ & $m_{y}$ & $\rho$ & $s_{x}$ & $s_{y}$ & $\Delta E$ \\
\hline Exact solution & & 1.125 & 1.125 & 0 & 0.9 & 0.9 & 0.85 \\
\hline \multirow{4}{*}{ Relative error 1\% } & TRVF & 1.1238 & 1.1251 & -0.0007 & 0.8983 & 0.8966 & 0.8515 \\
& Error (\%) & $\mathbf{- 0 . 1}$ & $\mathbf{0 . 0 1}$ & $\mathbf{- 0 . 0 7}$ & $\mathbf{- 0 . 1 9}$ & $\mathbf{- 0 . 3 8}$ & $\mathbf{0 . 1 8}$ \\
\cline { 2 - 8 } & Original & 1.1192 & 1.1257 & -0.0017 & 0.8937 & 0.8959 & 0.8523 \\
& Error (\%) & -0.51 & 0.06 & -0.17 & -0.7 & -0.45 & 0.27 \\
\hline \multirow{5}{*}{ Relative error 5\% } & TRVF & 1.1172 & 1.1246 & -0.0013 & 0.8887 & 0.8853 & 0.859 \\
& Error (\%) & $\mathbf{- 0 . 7}$ & $\mathbf{- 0 . 0 4}$ & $\mathbf{- 0 . 1 3}$ & $\mathbf{- 1 . 2 6}$ & $\mathbf{- 1 . 6 3}$ & $\mathbf{1 . 0 6}$ \\
\cline { 2 - 8 } & Original & 1.101 & 1.1284 & -0.0076 & 0.873 & 0.8821 & 0.8623 \\
& Error (\%) & -2.13 & 0.3 & -0.76 & -3 & -1.99 & 1.45 \\
\hline \multirow{5}{*}{ Relative error 10\% } & TRVF & 1.1082 & 1.1237 & -0.0041 & 0.8691 & 0.8806 & 0.8679 \\
& Error (\%) & $\mathbf{- 1 . 5}$ & $\mathbf{- 0 . 1 1}$ & -0.41 & $\mathbf{- 3 . 4 4}$ & -2.16 & 2.1 \\
\cline { 2 - 8 } & Original & 1.0866 & 1.1347 & -0.0029 & 0.862 & 0.8821 & 0.8678 \\
& Error (\%) & -3.41 & 0.86 & $\mathbf{- 0 . 2 9}$ & -4.22 & $\mathbf{- 1 . 9 9}$ & $\mathbf{2 . 0 9}$ \\
\hline
\end{tabular}

uniqueness of information in the bi-objective with $\alpha=1$ solution depicted in Figure 5(a) resulted in the best initial performance (after 15 iterations) with a similar sensor layout to the $\alpha=0.5$ solution depicted in Figure 5(b). It is again evident that uniqueness of information predominantly drives the solution to the inverse problem in the first 15 iterations.

Solutions for $\alpha=0.5$ and $\alpha=1$ both outperform the originally placed sensors by Lee and Song (2016) as quantified in Table 4. Note that Table 4 reports the number of iterations (\# iter.), function evaluations (\# f.e.) and difference norm $\|\mathbf{F}(E)\|)$ for the various sensor placements using three levels of noise for Scenario 2. Considering the converged Optimisation results as opposed to only after 15 iterations it is evident that both uniqueness of information and variance $(\alpha=0.5)$ are critical to consider, outperforming all solutions for all levels of noise. The $\alpha=0.5$ solution outperforms the original sensor layout solution and the next best solution by more than a factor of 2 and 1.2 , respectively, for $1 \%$ noise. This also implies that both uniqueness of information and variance drive the solution of the inverse problem towards the end of an optimisation run. As expected the difference between optimised and originally placed sensors decreases as the noise increases, as it is clear that all sensor layouts are equal when only noise is present.

Figure 6 depicts the $E(x, y)$ contours for various amounts of noise when 
Table 4: Scenario 2: inverse problem solutions using the sensor layouts for TRVF for $\alpha=1,0.5$ and 0 against the original sensor placement for different levels of stochastic noise.

\begin{tabular}{|c|c|c|c|c|c|c|c|c|c|}
\hline \multirow[b]{2}{*}{ Noise } & \multirow[b]{2}{*}{ Method } & \multicolumn{4}{|c|}{ Initial optimisation performance (after 15 iter.) } & \multicolumn{4}{|c|}{ Converged optimisation performance } \\
\hline & & \# iter. & \# f.e. & $\|\mathbf{F}(E)\|$ & $\%$ f.v. diff. & \# iter. & \# f.e. & $\|\mathbf{F}(E)\|$ & $\%$ f.v. diff \\
\hline \multirow{4}{*}{$1 \%$} & $\alpha=1$ & 15 & 136.2 & 627.2 & -85.4 & 90 & 850.2 & 171.7 & -94.2 \\
\hline & $\alpha=0.5$ & 15 & 134.9 & 770.1 & -85.2 & 91.3 & 859.4 & 142.8 & -95.1 \\
\hline & $\alpha=0$ & 15 & 137.7 & 1838.4 & -91.7 & 128.4 & 1102.2 & 957.3 & -94.2 \\
\hline & Original & 15 & 137.7 & 1064.2 & -86 & 87.6 & 805.9 & 321 & -93.8 \\
\hline \multirow{4}{*}{$5 \%$} & $\alpha=1$ & 15 & 134.3 & 738.6 & -75 & 78.2 & 749.9 & 447.8 & -78.6 \\
\hline & $\alpha=0.5$ & 15 & 132.7 & 799 & -75.3 & 85 & 812 & 415.9 & -79.9 \\
\hline & $\alpha=0$ & 15 & 138.3 & 2230.2 & -72.2 & 138.1 & 1206.9 & 1732.3 & -73.7 \\
\hline & Original & 15 & 134.8 & 1105.5 & -73.8 & 95 & 886.9 & 611.3 & -76.9 \\
\hline \multirow{4}{*}{$10 \%$} & $\alpha=1$ & 15 & 133.8 & 1018 & -56 & 90 & 848.6 & 837.5 & -59.2 \\
\hline & $\alpha=0.5$ & 15 & 134.5 & 1023 & -59.4 & 85.4 & 814.6 & 823.7 & -62.6 \\
\hline & $\alpha=0$ & 15 & 138.6 & 2204.3 & -48 & 137.1 & 1239.5 & 2066.4 & -49.3 \\
\hline & Original & 15 & 135.6 & 1287.8 & -50.4 & 101.5 & 966 & 1108.1 & -52.6 \\
\hline
\end{tabular}

solving TRVF using $\alpha=0.5$ and the original sensor layout (Lee and Song (2016)) for the converged solutions. The converged optimised results clearly indicate that TRVF sensor layout outperforms the original sensor placement of Lee and Song (2016).

Table 5 quantifies the differences in the optimal parameter values $\mathbf{w}$ when solving the inverse problem using the TRVF sensor layout and the original sensor layout by Lee and Song (2016) to the actual parameter values for the parametrisation of $E(x, y)$ as tabulated in Table 1 . This effectively quantifies the uniqueness of the parametrisation of $E(x, y)$ and allows for a comparison between the different solutions. Ultimately, the errors as tabulated in Table 4 are of primary concern.

\subsubsection{Scenario 3}

The deterioration field for Scenario 3 is at the lower left corner as shown in Figures 7(a)-(d). The optimised sensor locations are indicated as red dots for choices of $\alpha=1,0.5,0$ respectively in Figures 7(a)-(c), while the originally placed sensor locations are indicated in Figure $7(\mathrm{~d})$. The results are for $1 \%$ noise added to the sensor measurement and only after 15 iterations of solving the inverse problem. It is evident from Figure 7(c) that maximising variance on its own does not perform well with the sensors clustering around the left 


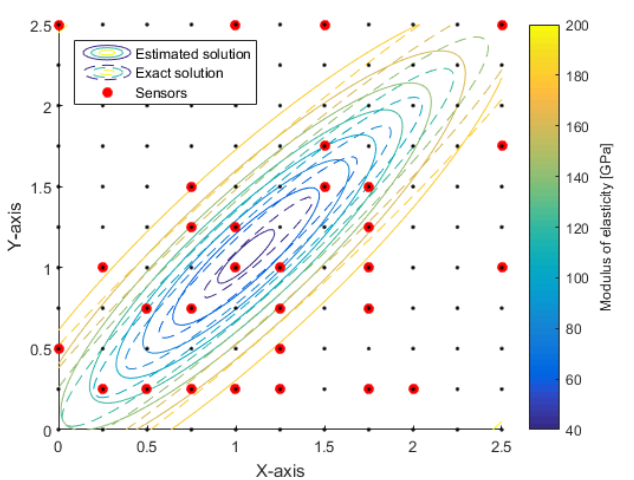

(a) TRVF $\alpha=1$

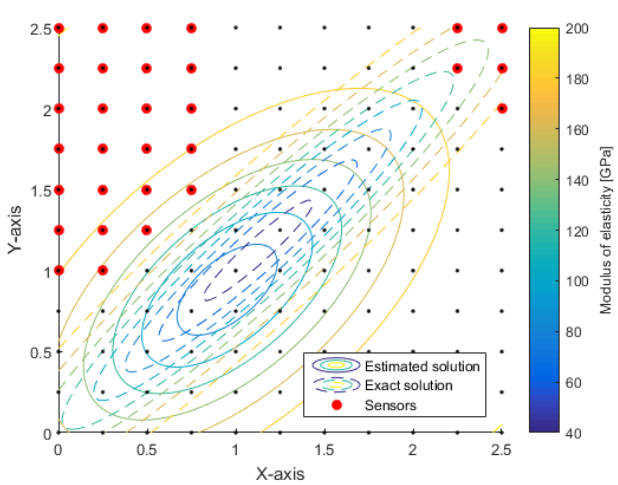

(c) TRVF $\alpha=0$

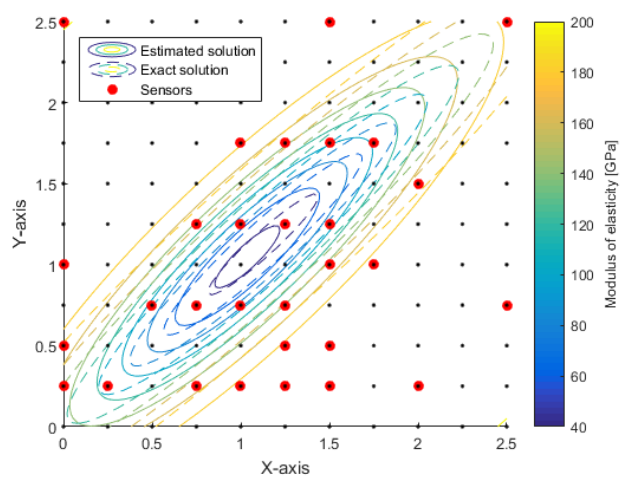

(b) TRVF $\alpha=0.5$

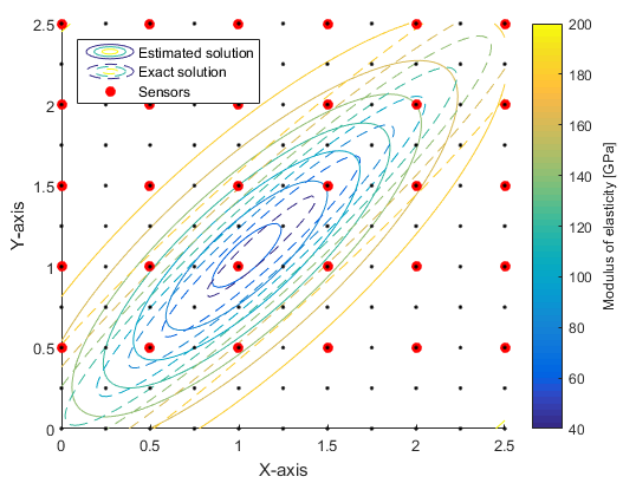

(d) Original sensor placements

Figure 5: Scenario 2: optimal sensor locations obtained by solving TRVF $(\alpha=1,0.5,0)$ presented respectively in (a),(b) and (c). In addition, (d) the sensor placement used by Lee and Song (2016). Depicted is the solution after 15 iterations of solving the inverse problem to characterise the deterioration contours of $E(x, y)$ with $1 \%$ of point noise on the measurements. 


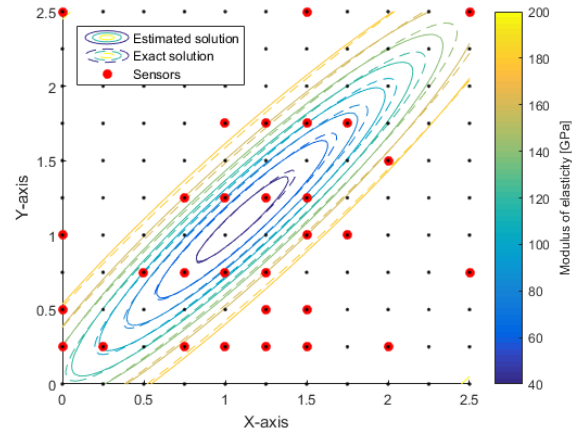

(a) Optimised sensors with $1 \%$ noise

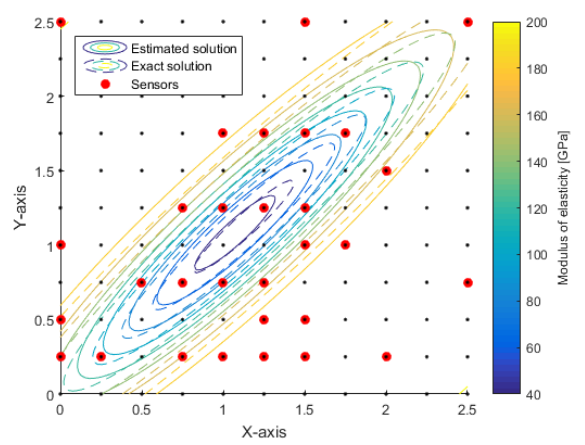

(c) Optimised sensors with $5 \%$ noise

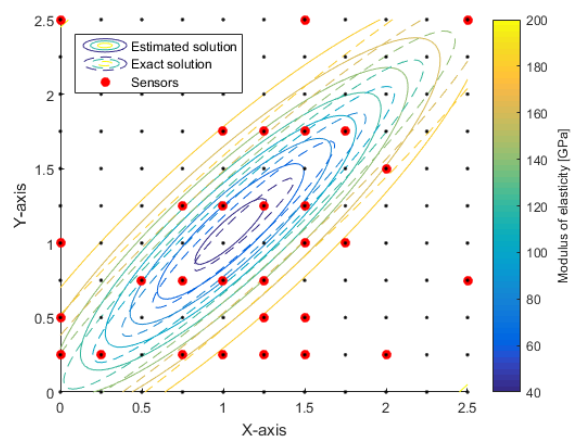

(e) Optimised sensors with $10 \%$ noise

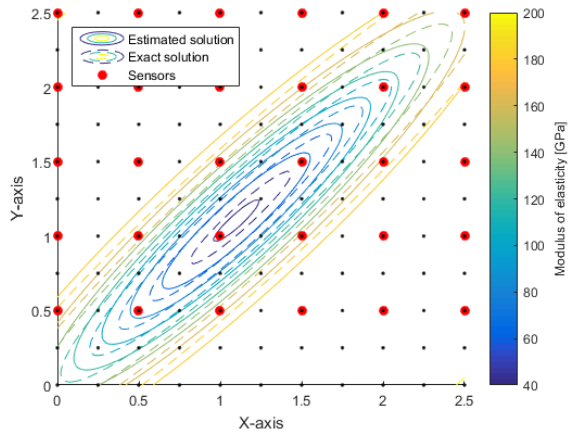

(b) Original sensors with $1 \%$ noise

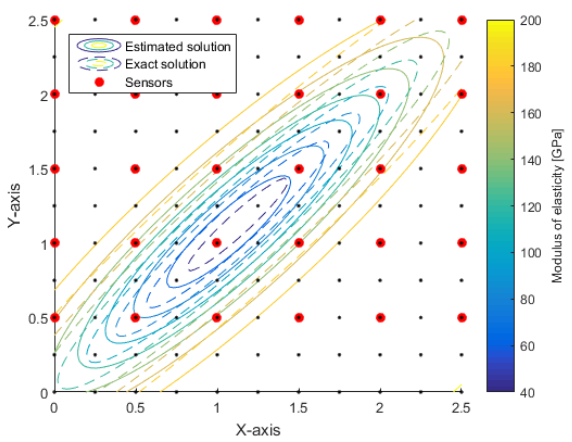

(d) Original sensors with 5\% noise

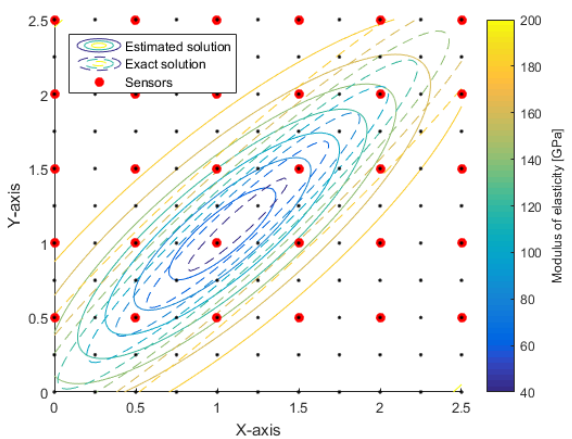

(f) Original sensors with $10 \%$ noise

Figure 6: Scenario 2: optimal deterioration contours of $E(x, y)$ after solving the inverse problem to convergence using the optimal sensor placement obtained by solving TRVF ( $\alpha$ $=0.5$ ) versus the sensor placement used by Lee and Song (2016) with (a),(b) 1\%, (c),(d) $5 \%$ and (e),(f) $10 \%$ relative point noise on the measurements. 
Table 5: Scenario 2: parameter errors for TRVF $(\alpha=0.5)$ and the original sensor placement by Lee and Song (2016) against the known solution as tabulated in Table 1.

\begin{tabular}{llllllll}
\hline Scenario 2 & & $m_{x}$ & $m_{y}$ & $\rho$ & $s_{x}$ & $s_{y}$ & $\Delta E$ \\
\hline Exact solution & & 1.125 & 1.125 & 0.95 & 0.9 & 0.9 & 0.85 \\
\hline \multirow{3}{*}{ Relative error 1\% } & TRVF & 1.1071 & 1.1045 & 0.94 & 0.8614 & 0.8562 & 0.8456 \\
& Error (\%) & $\mathbf{- 1 . 5 9}$ & $\mathbf{- 1 . 8 3}$ & $\mathbf{- 1 . 0 6}$ & $\mathbf{- 4 . 2 9}$ & $\mathbf{- 4 . 8 7}$ & $\mathbf{- 0 . 5 1}$ \\
\cline { 2 - 8 } & Original & 1.1028 & 1.0983 & 0.9238 & 0.8422 & 0.8384 & 0.8117 \\
& Error (\%) & -1.98 & -2.38 & -2.76 & -6.42 & -6.85 & -4.51 \\
\hline \multirow{3}{*}{ Relative error 5\% } & TRVF & 1.0834 & 1.0714 & 0.9157 & 0.8034 & 0.7934 & 0.8403 \\
& Error (\%) & $\mathbf{- 3 . 6 9}$ & $\mathbf{- 4 . 7 7}$ & $\mathbf{- 3 . 6 1}$ & $\mathbf{- 1 0 . 7 3}$ & -11.84 & $\mathbf{- 1 . 1 4}$ \\
\cline { 2 - 8 } & Original & 1.0764 & 1.0705 & 0.8902 & 0.7895 & 0.7987 & 0.7843 \\
& Error (\%) & -4.32 & -4.85 & -6.3 & -12.28 & $\mathbf{- 1 1 . 2 6}$ & $\mathbf{- 7 . 7 2}$ \\
\hline \multirow{2}{*}{ Relative error 10\% } & TRVF & 1.079 & 1.0457 & 0.8831 & 0.7687 & 0.7611 & 0.8343 \\
& Error (\%) & $\mathbf{- 4 . 0 9}$ & $\mathbf{- 7 . 0 5}$ & $\mathbf{- 7 . 0 5}$ & $\mathbf{- 1 4 . 5 8}$ & -15.44 & $\mathbf{- 1 . 8 5}$ \\
\cline { 2 - 7 } & Original & 1.062 & 1.0314 & 0.832 & 0.7354 & 0.7803 & 0.765 \\
& Error (\%) & -5.6 & -8.32 & -12.42 & -18.29 & $\mathbf{- 1 3 . 3}$ & $\mathbf{- 1 0}$ \\
\hline
\end{tabular}

edge of the plate. However, interestingly, it outperformed the $\alpha=1$ solution which looks almost identical to the $\alpha=0.5$ solution which outperformed both $\alpha=1$ and 0 by more than a factor of 2 for $1 \%$ noise (see Table 6 ). In fact, the original sensor layout outperforms the three $\alpha$ cases only after 15 iterations although it is evident that both $\alpha=1$ and 0.5 outperform the original sensor solutions almost by a factor of 3 for $1 \%$ noise when considering the converged optimisation results. As expected the difference between optimised and originally placed sensors decreases as the noise increases. Note that the converged results of both $\alpha=0.5$ and 0 are very close to each other. This illustrates that in some cases, the sensors chosen for maximising variance in sensor measurements may result in unique information as sensors are spatially distinct by placement. Figure 8 compares the $E(x, y)$ recovered using TRVF versus the original sensor layout. It is observed that estimated solution contour of the TRVF is much more accurate than that of the original sensors. Note that the optimised sensors are closely located around the deterioration area.

Table 7 quantifies the differences in the optimal parameter values w when solving the inverse problem using the TRVF sensor layout and the original sensor layout by Lee and Song (2016) to the actual parameter values for the parametrisation of $E(x, y)$ as tabulated in Table 1 . This effectively quantifies 
Table 6: Scenario 3: inverse problem solutions using the sensor layouts for TRVF for $\alpha=1,0.5$ and 0 against the original sensor placement for different levels of stochastic noise.

\begin{tabular}{|c|c|c|c|c|c|c|c|c|c|}
\hline \multirow[b]{2}{*}{ Noise } & \multirow[b]{2}{*}{ Method } & \multicolumn{4}{|c|}{ Initial optimisation performance (after 15 iter.) } & \multicolumn{4}{|c|}{ Converged optimisation performance } \\
\hline & & \# iter. & \# f.e. & $\|\mathbf{F}(E)\|$ & $\%$ f.v. diff. & \# iter. & \# f.e. & $\|\mathbf{F}(E)\|$ & $\%$ f.v. diff. \\
\hline \multirow{4}{*}{$1 \%$} & $\alpha=1$ & 15 & 123 & 1797.2 & -85.1 & 126.9 & 1112.2 & 111.9 & -97.4 \\
\hline & $\alpha=0.5$ & 15 & 128.2 & 678.8 & -91.4 & 115.4 & 1024 & 103.8 & -95.9 \\
\hline & $\alpha=0$ & 15 & 137.4 & 1367 & -93.4 & 213.3 & 1807.8 & 683 & -94.9 \\
\hline & Original & 15 & 133.9 & 604.4 & -90.2 & 121.9 & 1073.5 & 410.9 & -93.5 \\
\hline \multirow{4}{*}{$5 \%$} & $\alpha=1$ & 15 & 125.9 & 1737.6 & -80.6 & 149.1 & 1309.4 & 277.4 & -88 \\
\hline & $\alpha=0.5$ & 15 & 128.6 & 807.4 & -78.4 & 142.2 & 1259.1 & 272.8 & -81 \\
\hline & $\alpha=0$ & 15 & 133.5 & 1388.3 & -74.7 & 223.1 & 1975.5 & 943.7 & -76.2 \\
\hline & Original & 15 & 132.4 & 692.6 & -69.2 & 139.2 & 1250.3 & 544.1 & -71.3 \\
\hline \multirow{4}{*}{$10 \%$} & $\alpha=1$ & 15 & 128.3 & 1579.6 & -71.1 & 139 & 1230.5 & 517 & -75.3 \\
\hline & $\alpha=0.5$ & 15 & 132.6 & 958 & -63.1 & 138.2 & 1238.5 & 505.2 & -65.3 \\
\hline & $\alpha=0$ & 15 & 133.6 & 1413.9 & -50.6 & 176.7 & 1522.6 & 1101.5 & -52.8 \\
\hline & Original & 15 & 133.8 & 783.6 & -47.2 & 120.6 & 1076.9 & 624.6 & -49.4 \\
\hline
\end{tabular}

the uniqueness of the parametrisation of $E(x, y)$ and allows for a comparison between the different solutions. Ultimately, the errors as tabulated in Table 6 are of primary concern.

\subsubsection{Scenario 4}

Scenario 4 has its deterioration area around the top right corner of the plate as shown in Figure 9. It is evident from the figure that the optimised sensors with $\alpha=1,0.5$ and 0 which are closely located to the deterioration area outperform the original sensor layout result for $1 \%$ noise when only 15 iterations are considered. Table 8 shows that the results for $\alpha=0.5$, which considers both the uniqueness of information and variance, produce the lowest error $\|\mathbf{F}(E)\|$ after 15 optimisation iterations as well as the converged optimisation solution for all the noise settings. Figure 10 again illustrates that the optimised sensors recover the more accurate deterioration contours compared to the original sensor layout. Although the overall errors $\|\mathbf{F}(E)\|$ of TRVF $(\alpha=0)$ are less than those of the original sensor layout, each individual parameter error can be sometimes greater as shown in Table 9 .

The example study with the four scenarios clearly demonstrated that calibrating the contributions of rank tolerance $\epsilon_{r}$ and variance $v_{x}$ by changing the $\alpha$ value affects the near optimal placement of sensors. This also illustrated 


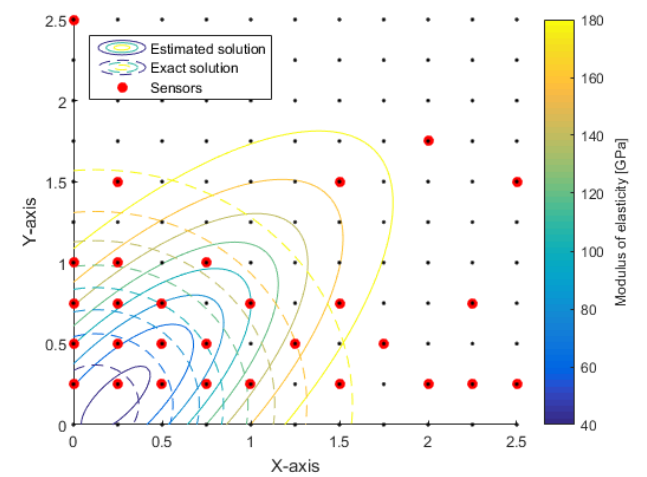

(a) TRVF $\alpha=1$

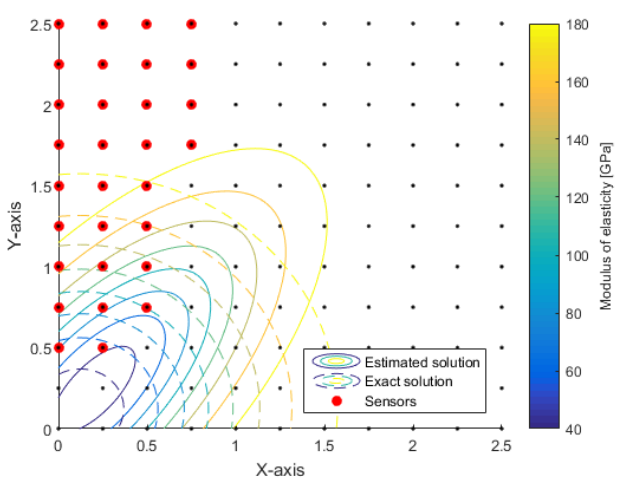

(c) TRVF $\alpha=0$

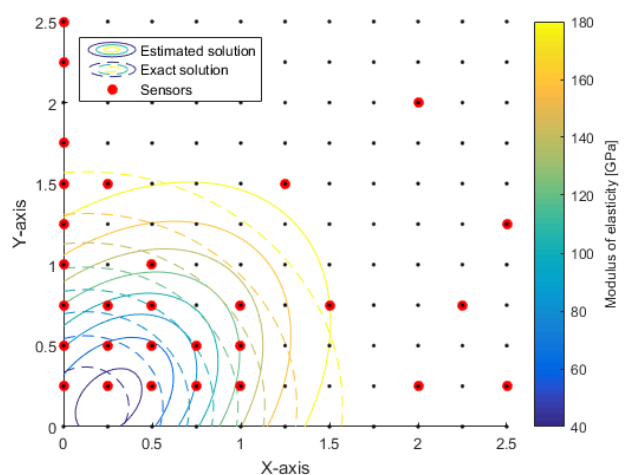

(b) TRVF $\alpha=0.5$

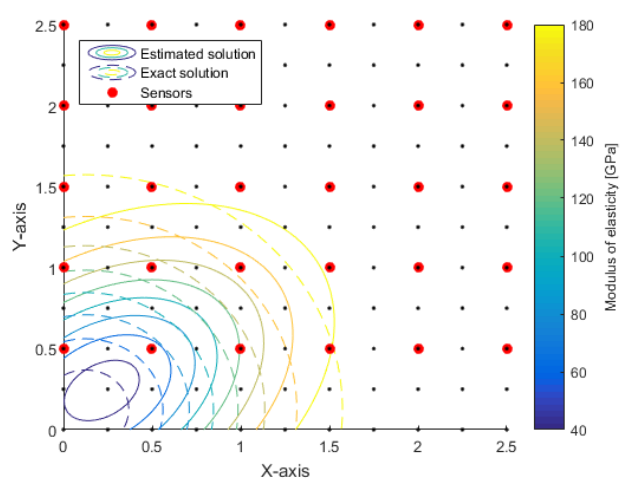

(d) Original sensor placements

Figure 7: Scenario 3: optimal sensor locations obtained by solving TRVF $(\alpha=1,0.5,0)$ presented respectively in (a),(b) and (c). In addition, (d) the sensor placement used by Lee and Song (2016). Depicted is the solution after 15 iterations of solving the inverse problem to characterise the deterioration contours of $E(x, y)$ with $1 \%$ of point noise on the measurements. 


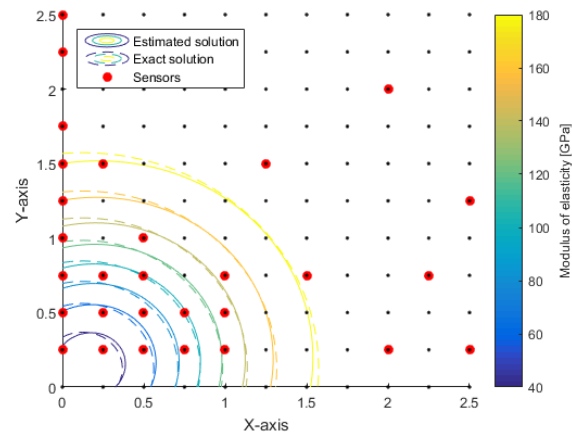

(a) Optimised sensors with $1 \%$ noise

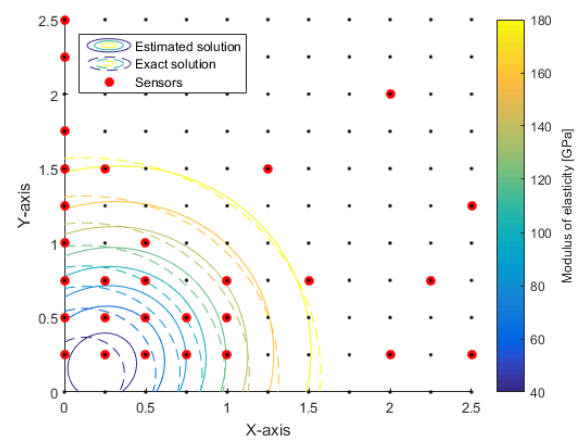

(c) Optimised sensors with 5\% noise

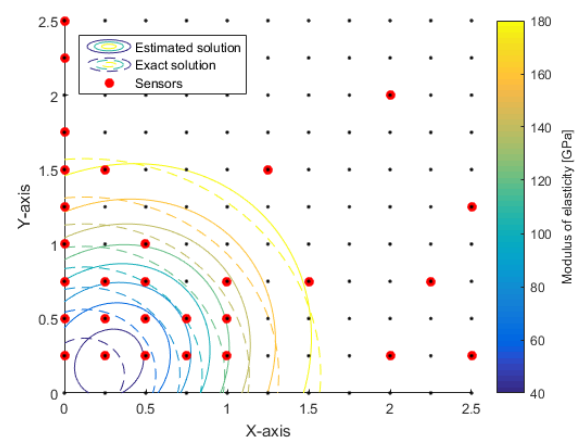

(e) Optimised sensors with $10 \%$ noise

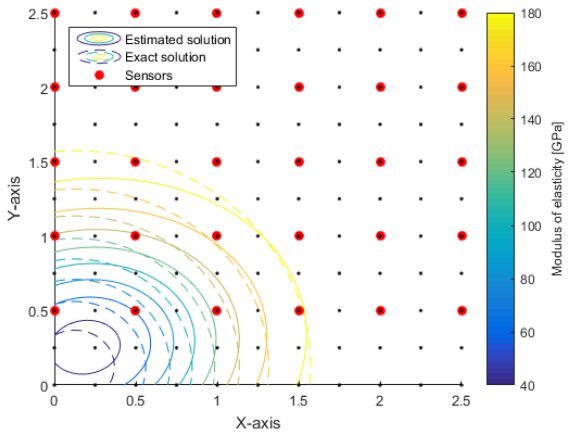

(b) Original sensors with $1 \%$ noise

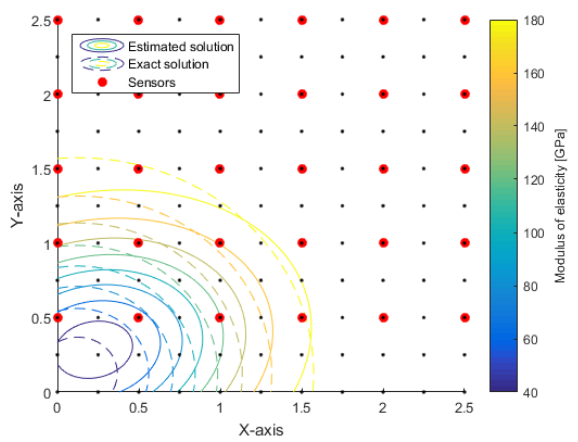

(d) Original sensors with $5 \%$ noise

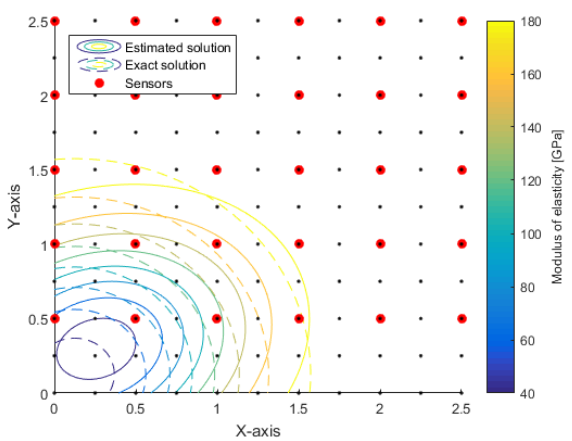

(f) Original sensors with $10 \%$ noise

Figure 8: Scenario 3: optimal deterioration contours of $E(x, y)$ after solving the inverse problem to convergence using the optimal sensor placement obtained by solving TRVF ( $\alpha$ $=0.5$ ) versus the sensor placement used by Lee and Song (2016) with (a),(b) 1\%, (c),(d) $5 \%$ and (e),(f) $10 \%$ point noise on the measurements. 
Table 7: Scenario 3: parameter errors for TRVF $(\alpha=0.5)$ and the original sensor placement by Lee and Song (2016) against the known solution as tabulated in Table 1.

\begin{tabular}{llllllll}
\hline Scenario 3 & & $m_{x}$ & $m_{y}$ & $\rho$ & $s_{x}$ & $s_{y}$ & $\Delta E$ \\
\hline Exact solution & & 0.125 & 0.125 & 0 & 0.7 & 0.7 & 0.85 \\
\hline \multirow{3}{*}{ Relative error 1\% } & TRVF & 0.1463 & 0.1691 & 0.0373 & 0.6642 & 0.6628 & 0.8446 \\
& Error (\%) & $\mathbf{1 7 . 0 4}$ & $\mathbf{3 5 . 2 5}$ & $\mathbf{3 . 7 3}$ & $\mathbf{- 5 . 1 1}$ & $\mathbf{- 5 . 3 1}$ & -0.64 \\
\cline { 2 - 8 } & Original & 0.2539 & 0.1876 & 0.0745 & 0.5493 & 0.6586 & 0.8445 \\
& Error (\%) & 103.09 & 50.08 & 7.45 & -21.52 & -5.91 & -0.64 \\
\hline \multirow{5}{*}{ Relative error 5\% } & TRVF & 0.1747 & 0.2322 & 0.0765 & 0.6492 & 0.6195 & 0.8481 \\
& Error (\%) & $\mathbf{3 9 . 8}$ & 85.75 & $\mathbf{7 . 6 5}$ & $\mathbf{- 7 . 2 5}$ & $\mathbf{- 1 1 . 5}$ & $\mathbf{- 0 . 2 3}$ \\
\cline { 2 - 8 } & Original & 0.284 & 0.2223 & 0.1381 & 0.5175 & 0.6449 & 0.8573 \\
& Error (\%) & 127.2 & $\mathbf{7 7 . 8 4}$ & 13.81 & $\mathbf{- 2 6 . 0 7}$ & $\mathbf{- 7 . 8 7}$ & 0.86 \\
\hline \multirow{3}{*}{ Relative error 10\% } & TRVF & 0.201 & 0.2743 & 0.1287 & 0.6469 & 0.6004 & 0.8515 \\
& Error (\%) & $\mathbf{6 0 . 8 1}$ & 119.43 & $\mathbf{1 2 . 8 7}$ & $\mathbf{- 7 . 5 9}$ & $\mathbf{- 1 4 . 2 3}$ & $\mathbf{0 . 1 8}$ \\
\cline { 2 - 7 } & Original & 0.2969 & 0.2563 & 0.1785 & 0.532 & 0.6309 & 0.8618 \\
& Error (\%) & 137.51 & $\mathbf{1 0 5 . 0 6}$ & 17.85 & $\mathbf{- 2 4}$ & $\mathbf{- 9 . 8 8}$ & 1.38 \\
\hline
\end{tabular}

Table 8: Scenario 4: inverse problem solutions using the sensor layouts for TRVF for $\alpha=1,0.5$ and 0 against the original sensor placement for different levels of stochastic noise.

\begin{tabular}{|c|c|c|c|c|c|c|c|c|c|}
\hline \multirow[b]{2}{*}{ Noise } & \multirow[b]{2}{*}{ Method } & \multicolumn{4}{|c|}{ Initial optimisation performance (after 15 iter.) } & \multicolumn{4}{|c|}{ Converged optimisation performance } \\
\hline & & \# iter. & \# f.e. & $\|\mathbf{F}(E)\|$ & $\%$ f.v. diff. & \# iter. & \# f.e. & $\|\mathbf{F}(E)\|$ & $\%$ f.v. diff. \\
\hline \multirow{4}{*}{$1 \%$} & $\alpha=1$ & 15 & 129.4 & 217.6 & -90.2 & 98.7 & 897.2 & 264.4 & -92.1 \\
\hline & $\alpha=0.5$ & 15 & 127.3 & 162.8 & -90 & 110.1 & 1011.1 & 225.9 & -92.8 \\
\hline & $\alpha=0$ & 15 & 125.4 & 214.3 & -90.1 & 113.3 & 1024.8 & 243.6 & -92.7 \\
\hline & Original & 15 & 132.1 & 608.6 & -86.9 & 124.7 & 1129.2 & 296.1 & -92.7 \\
\hline \multirow{4}{*}{$5 \%$} & $\alpha=1$ & 15 & 131.6 & 485.6 & -66 & 132.7 & 1188.2 & 650.4 & -69.1 \\
\hline & $\alpha=0.5$ & 15 & 128.2 & 460.6 & -64.2 & 118.4 & 1062 & 457.4 & -67.9 \\
\hline & $\alpha=0$ & 15 & 128.1 & 710.1 & -65 & 139.5 & 1261.5 & 487.7 & -68.8 \\
\hline & Original & 15 & 133 & 579.2 & -65.9 & 129.6 & 1169.1 & 575 & -69.6 \\
\hline \multirow{4}{*}{$10 \%$} & $\alpha=1$ & 15 & 133.5 & 916.1 & -46.2 & 136.1 & 1207.3 & 939 & -49.2 \\
\hline & $\alpha=0.5$ & 15 & 130.7 & 798.2 & -44.4 & 135.3 & 1205.2 & 780.7 & -47.7 \\
\hline & $\alpha=0$ & 15 & 128.1 & 1135.9 & -44.8 & 137.7 & 1219.8 & 986.5 & -48.5 \\
\hline & Original & 15 & 131.4 & 1064.4 & -45.2 & 126.3 & 1125 & 1047.6 & -48.7 \\
\hline
\end{tabular}




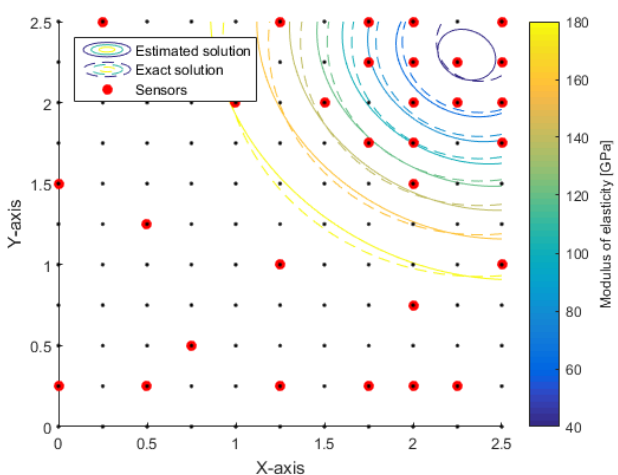

(a) TRVF $\alpha=1$

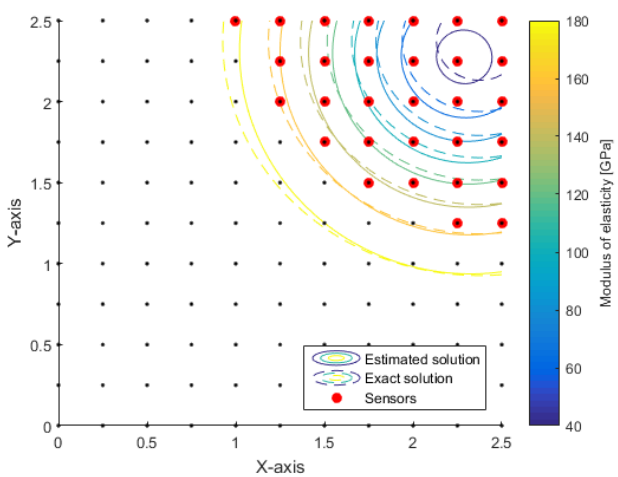

(c) TRVF $\alpha=0$

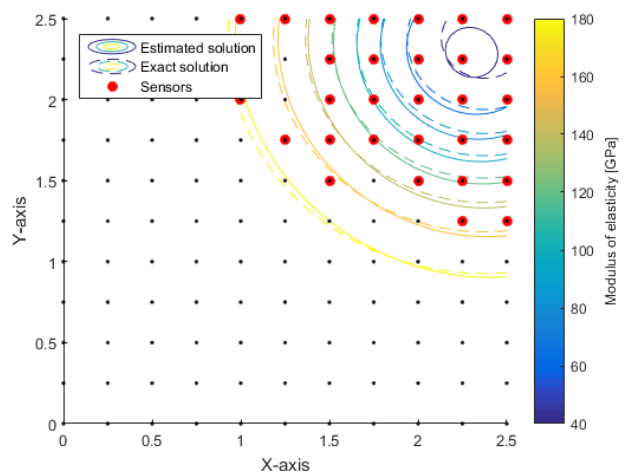

(b) TRVF $\alpha=0.5$

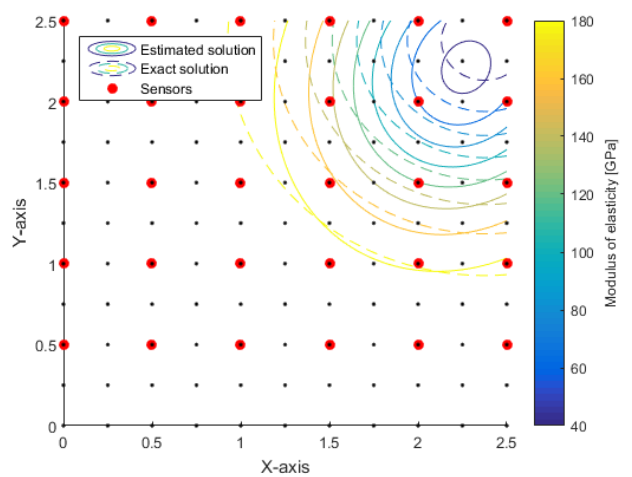

(d) Original sensor placements

Figure 9: Scenario 4: optimal sensor locations obtained by solving TRVF $(\alpha=1,0.5,0)$ presented respectively in (a),(b) and (c). In addition, (d) the sensor placement used by Lee and Song (2016). Depicted is the solution after 15 iterations of solving the inverse problem to characterise the deterioration contours of $E(x, y)$ with $1 \%$ of point noise on the measurements. 


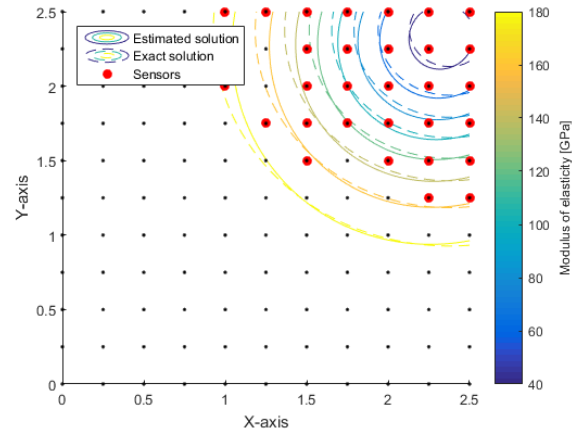

(a) Optimised sensors with $1 \%$ noise

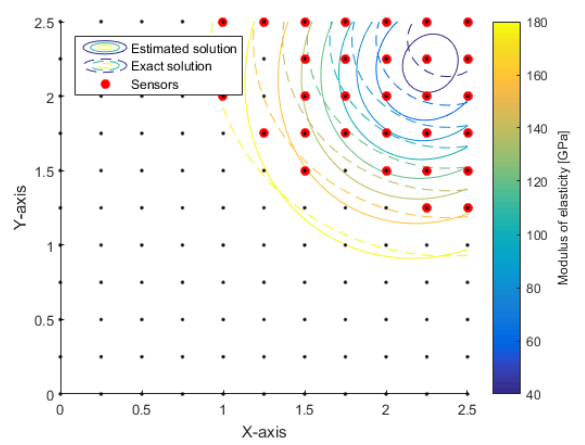

(c) Optimised sensors with $5 \%$ noise

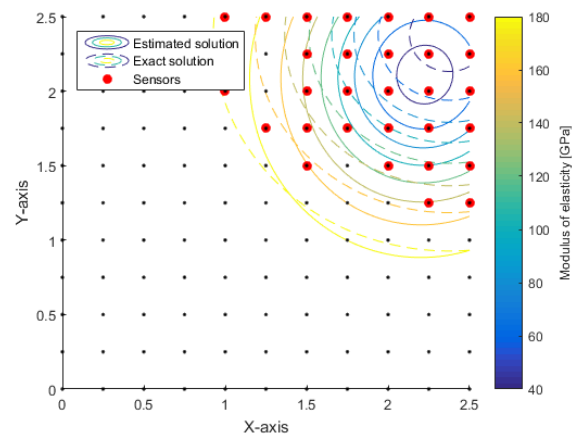

(e) Optimised sensors with $10 \%$ noise

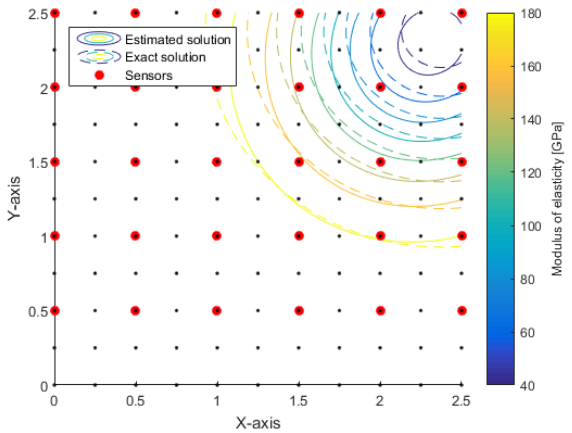

(b) Original sensors with $1 \%$ noise

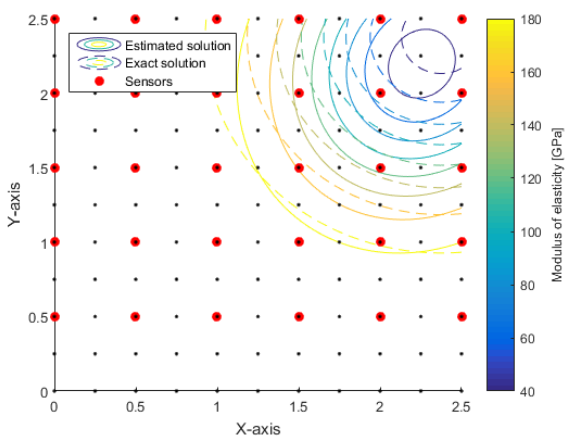

(d) Original sensors with $5 \%$ noise

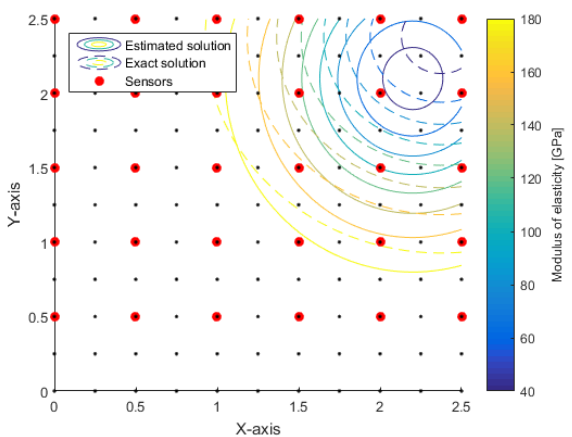

(f) Original sensors with $10 \%$ noise

Figure 10: Scenario 4: optimal deterioration contours of $E(x, y)$ after solving the inverse problem to convergence using the optimal sensor placement obtained by solving TRVF ( $\alpha$ $=0.5$ ) versus the sensor placement used by Lee and Song (2016) with (a),(b) 1\%, (c),(d) $5 \%$ and (e),(f) $10 \%$ point noise on the measurements. 
Table 9: Scenario 4: parameter errors for TRVF $(\alpha=0.5)$ and the original sensor placement by Lee and Song (2016) against the known solution as tabulated in Table 1.

\begin{tabular}{llllllll}
\hline Scenario 4 & & $m_{x}$ & $m_{y}$ & $\rho$ & $s_{x}$ & $s_{y}$ & $\Delta E$ \\
\hline Exact solution & & 2.375 & 2.375 & 0 & 0.7 & 0.7 & 0.85 \\
\hline \multirow{4}{*}{ Relative error 1\% $\%$} & TRVF & 2.3295 & 2.3278 & 0.0525 & 0.674 & 0.6232 & 0.8423 \\
& Error (\%) & $\mathbf{- 1 . 9 2}$ & $\mathbf{- 1 . 9 9}$ & $\mathbf{5 . 2 5}$ & $\mathbf{- 3 . 7 1}$ & $\mathbf{- 1 0 . 9 7}$ & $\mathbf{- 0 . 9 1}$ \\
\cline { 2 - 8 } & Original & 2.3043 & 2.3155 & 0.1007 & 0.6499 & 0.6003 & 0.8488 \\
& Error (\%) & -2.98 & -2.5 & 10.07 & -7.16 & -14.25 & -0.15 \\
\hline \multirow{5}{*}{ Relative error 5\% } & TRVF & 2.2207 & 2.2714 & 0.0998 & 0.6348 & 0.5517 & 0.8393 \\
& Error (\%) & $\mathbf{- 6 . 5}$ & $\mathbf{- 4 . 3 6}$ & $\mathbf{9 . 9 8}$ & $\mathbf{- 9 . 3 1}$ & $\mathbf{- 2 1 . 1 8}$ & -1.26 \\
\cline { 2 - 8 } & Original & 2.1978 & 2.2562 & 0.1253 & 0.6132 & 0.5469 & 0.8588 \\
& Error (\%) & -7.46 & -5 & 12.53 & -12.41 & -21.88 & $\mathbf{1 . 0 4}$ \\
\hline \multirow{3}{*}{ Relative error 10\% } & TRVF & 2.1109 & 2.2252 & 0.0229 & 0.5941 & 0.5204 & 0.8455 \\
& Error (\%) & $\mathbf{- 1 1 . 1 2}$ & $\mathbf{- 6 . 3 1}$ & 2.29 & $\mathbf{- 1 5 . 1 2}$ & -25.66 & $\mathbf{- 0 . 5 3}$ \\
\cline { 2 - 8 } & Original & 2.0981 & 2.201 & 0.0003 & 0.6287 & 0.5573 & 0.8454 \\
& Error (\%) & -11.66 & -7.33 & $\mathbf{0 . 0 3}$ & $\mathbf{- 1 0 . 1 9}$ & $\mathbf{- 2 0 . 3 8}$ & -0.55 \\
\hline
\end{tabular}

that the near optimal placement of the sensors were highly dependent on the spatial deterioration patterns in the plates for exactly the same loading and boundary conditions. This clearly suggests that there is merit in considering spatially distributed variance as opposed to localised variance. Practically this also highlights the merits of instrumenting different parts of the structure according to expected deterioration which would depend on the loading of a component which differs between components in the same structure. It also demonstrates the importance of considering both rank tolerance and variance as opposed to only one of $\epsilon_{r}$ or $v_{x}$ ( $\alpha=1$ or 0 , respectively) which results in poor sensor placements whereas the $\alpha=0.5$ seems to be a robust first estimate. Even if the measurements obtained in a problem generally have high variance and maximising the $v_{r}$ may be negligible, one cannot always be certain whether that is true or not when facing the problem. Hence, the $\alpha$ of 0.5 can be chosen as an approximate value and it still outperforms the sensors placed simply with engineering intuition. 


\section{Conclusion}

A new sensor placement optimisation formulation, namely the tolerance rank-variance formulation (TRVF), is proposed. The formulation simply considers the rank of two covariance matrices and the variance information of the sensor sets towards the aim of solving inverse problems. In addition, a computationally efficient heuristic approach to solve both formulations are proposed and investigated numerically. It is not tractable to solve the formulations exhaustively as there are $8.3662 \times 10^{26}$ combinations that need to be evaluated for each of the four scenarios of material deterioration parametrisation problems considered in this study, in which the full spatial field deterioration of a plate is resolved. Although, no proof of optimality is offered for our proposed approach, the numerical results indicate quality solutions that are computationally tractable considering four scenarios. The four scenarios clearly demonstrated the that the near optimal positions of the sensors were highly dependent on the various shapes of the spatial deterioration patterns on the plates with exactly the same loading and boundary conditions. This clearly suggests that there is merit in instrumenting different parts of the structure according to expected deterioration which would depend on the loading of a component which differs between components in the same structure. It also demonstrates the importance of considering both rank tolerance and variance as opposed to only one of $\epsilon_{r}$ or $v_{x}$ ( $\alpha=1$ or 0 , respectively) which results in poor sensor placements whereas the $\alpha=0.5$ seems to be a robust first estimate.

\section{Acknowledgements}

This work was supported by the National Research Foundation (NRF), South Africa and Centre for Asset and Integrity Management (C-AIM), Department of Mechanical and Aeronautical Engineering, University of Pretoria, Pretoria, South Africa.

\section{References}

Achanta, H., Dasgupta, S. and Ding, Z. (2012). Optimum sensor placement for localization in three dimensional under log normal shadowing. 2012 5th International Congress on Image and Signal Processing. 
Asada, H. and Reisner, A. (2006). Wearable sensors for human health monitoring. Smart Structures and Materials 2006: Sensors and Smart Structures Technologies for Civil, Mechanical, and Aerospace Systems.

Beilina, L. (2015). Inverse problems and applications. Cham: Springer.

Bishop, C. (2013). Pattern recognition and machine learning. 1st ed. New York [u.a.]: Springer.

Bishop, A., Fidan, B., Anderson, B., Pathirana, P. and Dogancay, K. (2007). Optimality Analysis of Sensor-Target Geometries in Passive Localization: Part 2 - Time-of-Arrival Based Localization. 2007 3rd International Conference on Intelligent Sensors, Sensor Networks and Information.

Chae, Y. (2017). Optimal sensor placement approaches for the design fo inverse experiments by simulation, Master's degree, University of Pretoria.

Chisari, C., Macorini, L., Amadio, C. and Izzuddin, B. (2016). Optimal sensor placement for structural parameter identification. Structural and Multidisciplinary Optimization, 55(2), pp.647-662.

Dai, Y. and Li, H. (2014). Rank Minimization or Nuclear-Norm Minimization: Are We Solving the Right Problem?. 2014 International Conference on Digital Image Computing: Techniques and Applications (DICTA).

D'Andrea, E. and Marcelloni, F. (2017). Detection of traffic congestion and incidents from GPS trace analysis, Expert Systems with Applications, 73, pp.43-56.

Du, W., Xing, Z., Li, M., He, B., Chua, L. and Miao, H. (2014). Optimal sensor placement and measurement of wind for water quality studies in urban reservoirs. IPSN-14 Proceedings of the 13th International Symposium on Information Processing in Sensor Networks.

Du, W., Xing, Z., Li, M., He, B., Chua, L.H.C. and Miao, H. (2015). Sensor placement and measurement of wind for water quality studies in urban reservoirs, ACM Transactions on Sensor Networks, 11(3), pp.1-27.

Hadamard, J. (1902). Sur les problmes aux drives partielles et leur signification physique, Princeton University Bulletin, 13, pp.49-52. 
Helton, J.C., Davis, F.J. and Johnson, J.D. (2005). A comparison of uncertainty and sensitivity analysis results obtained with random and Latin hypercube sampling, Reliability Engineering 8 System Safety, 89(3), pp.305330 .

Fouladgar, J. (1997). The inverse problem methodology for the measurement of the permeability of the ferromagnetic materials. IEEE Transactions on Magnetics, 33(2), pp.2139-2142.

Isaacs, J., Klein, D. and Hespanha, J. (2009). Optimal sensor placement for time difference of arrival localization. Proceedings of the $48 \mathrm{~h}$ IEEE Conference on Decision and Control (CDC) held jointly with 2009 28th Chinese Control Conference.

Joshi, S. and Boyd, S. (2009). Sensor Selection via Convex Optimization. IEEE Transactions on Signal Processing, 57(2), pp.451-462.

Konak, A., Coit, D. and Smith, A. (2006). Multi-objective optimization using genetic algorithms: A tutorial. Reliability Engineering 85 System Safety, 91(9), pp.992-1007.

Korte, B. and Vygen, J. (2012). Combinatorial optimisation theory and algorithms. 5th edn. Heidelberg: Springer-Verlag Berlin Heidelberg.

Krause, A., Guestrin, C., Gupta, A. and Kleinberg, J. (2006). Near-optimal sensor placements: maximizing information while minimizing communication cost. 2006 5th International Conference on Information Processing in Sensor Networks.

Kreyszig, E. (1979). Advanced engineering mathematics. 4th edn. New York: New York Wiley 1979.

Lan, S., Qilong, M. and Du, J. (2008). Architecture of Wireless Sensor Networks for Environmental Monitoring. 2008 International Workshop on Education Technology and Training 62008 International Workshop on Geoscience and Remote Sensing.

Lee, S.-H. and Song, J. (2016). Bayesian-network-based system identification of spatial distribution of structural parameters, Engineering Structures, 127, pp.260-277. 
Li, Y., Xiang, Z., Zhou, M. and Cen, Z. (2007). An integrated parameter identification method combined with sensor placement design. Communications in Numerical Methods in Engineering, 24(12), pp.1571-1585.

Liu, B., Chen, X., Fang, D., Perrone, A., Pispas, S. and Vainos, N. (2010). Environmental monitoring by thin film nanocomposite sensors for cultural heritage preservation. Journal of Alloys and Compounds, 504, pp.S405S409.

MATLAB version 8.6.0.267246 (2015). computer software, The MathWorks Inc., Natick, Massachusetts.

Mungla, M.J., Sharma, D.S. and Trivedi, R.R. (2016). Inverse method to identify crack parameters in multi-span beam using genetic algorithm, Nondestructive Testing and Evaluation, pp.1-18.

Recht, B., Fazel, M. and Parrilo, P. (2010). Guaranteed Minimum-Rank Solutions of Linear Matrix Equations via Nuclear Norm Minimization. SIAM Review, 52(3), pp.471-501.

Reyes-Sierra, M. and Coello Coello, C. (2006). Dynamic fitness inheritance proportion for multi-objective particle swarm optimization. Proceedings of the 8th annual conference on Genetic and evolutionary computation GECCO 'O6.

Frieden, B. (2004). Science from Fisher information. Cambridge, England: Cambridge University Press.

Tang, B. (1993). Orthogonal Array-Based Latin Hypercubes. Journal of the American Statistical Association, 88(424), pp.1392-1397.

Van Trees, H.L. (1968). Detection, Estimation and Modulation Theory - Part 1: Detection, Estimation and Linear Modulation Theory. 1st ed. New York, NY: John Wiley and Sons, Inc.

Wang, W., Liu, X. and Liu, L. (2014). Upright orientation of 3D shapes via tensor rank minimization. Journal of Mechanical Science and Technology, 28(7), pp.2469-2477.

Wu, X. and Liu, M. (2012). In-situ soil moisture sensing. Proceedings of the 11th international conference on Information Processing in Sensor Networks - IPSN '12. 
Yao, L., Sethares, W. and Kammer, D. (1993). Sensor placement for onorbit modal identification via a genetic algorithm. AIAA Journal, 31(10), pp.1922-1928.

Yap, K. (2013). Efficient and Robust Solution of Inverse Structural Dynamic Problems for Vibration Health Monitoring. Topics in Modal Analysis, Volume 7, pp.155-166. 